TRANSACTIONS OF THE

AMERICAN MATHEMATICAL SOCIETY

Volume 363, Number 4, April 2011, Pages 1887-1924

S 0002-9947(2010)05200-6

Article electronically published on November 5, 2010

\title{
AFFINE AND QUASI-AFFINE FRAMES FOR RATIONAL DILATIONS
}

\author{
MARCIN BOWNIK AND JAKOB LEMVIG
}

\begin{abstract}
In this paper we extend the investigation of quasi-affine systems, which were originally introduced by Ron and Shen [J. Funct. Anal. 148 (1997), 408-447] for integer, expansive dilations, to the class of rational, expansive dilations. We show that an affine system is a frame if, and only if, the corresponding family of quasi-affine systems are frames with uniform frame bounds. We also prove a similar equivalence result between pairs of dual affine frames and dual quasi-affine frames. Finally, we uncover some fundamental differences between the integer and rational settings by exhibiting an example of a quasi-affine frame such that its affine counterpart is not a frame.
\end{abstract}

\section{INTRODUCTION}

Quasi-affine systems are little known cousins of well-studied affine systems also known as wavelet systems. Let $A$ be an expansive dilation matrix, i.e., an $n \times n$ real matrix with all eigenvalues $|\lambda|>1$. The affine system generated by a function $\psi \in L^{2}\left(\mathbb{R}^{n}\right)$ is

$$
\mathcal{A}(\psi)=\left\{\psi_{j, k}(x):=|\operatorname{det} A|^{j / 2} \psi\left(A^{j} x-k\right): j \in \mathbb{Z}, k \in \mathbb{Z}^{n}\right\} .
$$

The affine systems are dilation invariant, but not shift invariant. However, if the dilation $A$ has integer entries, that is $A \mathbb{Z}^{n} \subset \mathbb{Z}^{n}$, then one can modify the definition of affine systems to obtain shift invariant systems. This leads to the notion of a quasi-affine system

$$
\mathcal{A}^{q}(\psi)=\left\{\tilde{\psi}_{j, k}(x):=\left\{\begin{array}{l}
|\operatorname{det} A|^{j / 2} \psi\left(A^{j} x-k\right): j \geq 0, k \in \mathbb{Z}^{n} \\
|\operatorname{det} A|^{j} \psi\left(A^{j}(x-k)\right): j<0, k \in \mathbb{Z}^{n}
\end{array}\right\},\right.
$$

which was introduced and investigated for integer, expansive dilation matrices by Ron and Shen 20. Despite the fact that the orthogonality of the affine system cannot be carried over to the corresponding quasi-affine system due to the oversampling of negative scales of the affine system, it turns out that the frame property is preserved. This important discovery is due to Ron and Shen [20] who proved that the affine system $\mathcal{A}(\psi)$ is a frame if, and only if, its quasi-affine counterpart $\mathcal{A}^{q}(\psi)$ is a frame (with the same frame bounds). Furthermore, quasi-affine systems are

Received by the editors September 24, 2008.

2010 Mathematics Subject Classification. Primary 42C40.

Key words and phrases. Wavelets, affine systems, quasi-affine systems, rational dilations, shift invariant systems, oversampling.

The first author was partially supported by NSF grant DMS-0653881.

(C)2010 American Mathematical Society Reverts to public domain 28 years from publication 
shift invariant and thus much easier to study than affine systems which are dilation invariant.

The goal of this work is to extend the study of quasi-affine systems to the class of expansive rational dilations. Let $A$ be an expansive dilation with rational entries; that is, $A \mathbb{Q}^{n} \subset \mathbb{Q}^{n}$. The first author 3 generalized the notion of a quasi-affine frame for rational, expansive dilations which coincides with the usual definition in the case of integer dilations. The main idea of Ron and Shen [20] is to oversample negative scales of the affine system at a rate adapted to the scale in order for the resulting system to be shift invariant, i.e., $\phi \in \mathcal{A}^{q}(\psi) \Rightarrow T_{k} \phi \in \mathcal{A}^{q}(\psi)$ for all $k \in \mathbb{Z}^{n}$. In order to define quasi-affine systems for rational expansive dilations one needs to oversample both negative and positive scales of the affine system (at a rate proportional to the scale) which results in a quasi-affine system that in general coincides with the affine system only at the scale zero. This can easily be seen in one dimension where the quasi-affine system has a relatively simple algebraic form. Suppose that $a=p / q \in \mathbb{Q}$ is a dilation factor, where $|a|>1, p, q \in \mathbb{Z}$ are relatively prime. Then, the quasi-affine system associated with $a$ is given by

$$
\mathcal{A}^{q}(\psi)=\left\{\begin{array}{ll}
|p|^{j / 2}|q|^{-j} \psi\left(a^{j} x-q^{-j} k\right): & j \geq 0, k \in \mathbb{Z} \\
|p|^{j}|q|^{-j / 2} \psi\left(a^{j} x-p^{j} k\right): & j<0, k \in \mathbb{Z}
\end{array}\right\} .
$$

In the rational case it is much less clear than in the case of integer, expansive dilations (where both systems coincide at all non-negative scales), whether there is any relationship between affine and quasi-affine systems. Nevertheless, the first author proved in [3] that the tight frame property is preserved when moving between rationally dilated affine and quasi-affine systems. This result has initially suggested that there is not much difference between integer and rational cases.

In this work we show that this belief is largely incorrect by uncovering substantial differences between the theory of integer dilated and rationally dilated quasi-affine systems. For any rational, non-integer dilation we give an example of an affine system which is not a frame, but yet the corresponding quasi-affine system is a frame. This kind of example does not exist for integer dilations due to the above mentioned result of Ron and Shen.

To understand the broken symmetry between the integer and rational case we introduce a new class of quasi-affine systems indexed by the choice of the oversampling lattice $\Lambda \subset \mathbb{Z}^{n}$. In short, the quasi-affine system $\mathcal{A}_{\Lambda}^{q}(\psi)$ is defined to be the smallest shift invariant system with respect to a lattice $\Lambda$, i.e., $\phi \in \mathcal{A}_{\Lambda}^{q}(\psi) \Rightarrow T_{\lambda} \phi \in \mathcal{A}_{\Lambda}^{q}(\psi)$ for $\lambda \in \Lambda$, which contains all elements of the original affine system $\mathcal{A}(\psi)$. In order to make this definition meaningful we also need to renormalize the elements of $\mathcal{A}_{\wedge}^{q}(\psi)$ at a rate corresponding to the rate of oversampling as it was previously done. Again, this is best illustrated in one dimension. We take $\Lambda=(p q)^{J} \mathbb{Z}$ for $J \in \mathbb{N}_{0}$, since this particular choice gives the oversampled quasi-affine system $\mathcal{A}_{\Lambda}^{q}(\psi)$ a nice algebraic form:

$$
\mathcal{A}_{\Lambda}^{q}(\psi)=\left\{\begin{array}{ll}
|p|^{j / 2}|q|^{-j+J / 2} \psi\left(a^{j} x-q^{J-j} k\right): & j>J, k \in \mathbb{Z} \\
|a|^{j / 2} \psi\left(a^{j} x-k\right): & -J \leq j \leq J, k \in \mathbb{Z} \\
|p|^{j+J / 2}|q|^{-j / 2} \psi\left(a^{j} x-p^{j+J} k\right): & j<-J, k \in \mathbb{Z}
\end{array}\right\} ;
$$

see Example 3, Then our main result can be stated as follows. 
Theorem 1.1. The affine system $\mathcal{A}(\psi)$ is a frame for $L^{2}\left(\mathbb{R}^{n}\right)$ if, and only if, every $\Lambda$-oversampled quasi-affine system $\mathcal{A}_{\Lambda}^{q}(\psi)$ is a frame with uniform frame bounds for all $\wedge \subset \mathbb{Z}^{n}$.

In the case when the dilation $A$ is integer-valued, the class of $\Lambda$-oversampled quasi-affine systems reduces to the standard quasi-affine system $\mathcal{A}^{q}(\psi)$ and its dilates; see Example 2, Hence, the original result of Ron and Shen 20, follows immediately from Theorem 1.1. The proof of Theorem 1.1 is influenced by the work of Hernández, Labate, Weiss, and Wilson [13, 14, where the authors obtain reproducibility characterizations of generalized shift invariant (GSI) systems including affine, wave packets, and Gabor systems. The key element of these techniques is the use of almost periodic functions which was pioneered by Laugesen [17, 18] in his work on translational averaging of the wavelet functional. Using these methods Laugesen [18] gave another proof of the equivalence of affine and quasi-affine frames in the integer case. In this work we show that these techniques can be generalized to treat rationally dilated quasi-affine systems as well.

In the next part of the paper we investigate more subtle frame properties of quasiaffine systems. We characterize when the canonical dual frame of a $\Lambda$-oversampled quasi-affine frame $\mathcal{A}_{\Lambda}^{q}(\psi)$ is also a quasi-affine frame. In the case of integer dilations, such characterization is due to the first author and Weber [5]. Theorem 1.2 generalizes this result to the case of rational dilations. It is remarkable that the existence of the canonical quasi-affine dual frame is independent of the choice of the oversampling lattice $\Lambda$. Hence, if such canonical dual frame exists for some $\Lambda$-oversampled quasi-affine system, then it must exist for all lattices $\Lambda \subset \mathbb{Z}^{n}$.

Theorem 1.2. Suppose the quasi-affine system $\mathcal{A}_{\Lambda_{0}}^{q}(\psi)$ is a frame for $L^{2}\left(\mathbb{R}^{n}\right)$ for some lattice $\Lambda_{0} \subset \mathbb{Z}^{n}$. Then, the canonical dual frame of $\mathcal{A}_{\Lambda_{0}}^{q}(\psi)$ is of the form $\mathcal{A}_{\Lambda_{0}}^{q}(\phi)$ for some $\phi \in L^{2}\left(\mathbb{R}^{n}\right)$ if, and only if, for all $\alpha \in \mathbb{Z}^{n} \backslash\{0\}$,

$$
t_{\alpha}(\xi):=\sum_{j \in \mathbb{Z}: \alpha \in B^{j} \mathbb{Z}^{n}} \hat{\psi}\left(B^{-j} \xi\right) \overline{\hat{\psi}\left(B^{-j}(\xi+\alpha)\right)}=0 \quad \text { for a.e. } \xi \in \mathbb{R}^{n},
$$

where $B=A^{t}$. In this case, $\mathcal{A}_{\Lambda}^{q}(\phi)$ is the canonical dual frame of $\mathcal{A}_{\Lambda}^{q}(\psi)$ for all lattices $\wedge \subset \mathbb{Z}^{n}$.

We also investigate pairs of dual quasi-affine frames. Here, the theory of rationally dilated quasi-affine frames parallels quite closely that of integer dilated systems. Hence, we have a perfect equivalence between pairs of dual affine frames and pairs of dual quasi-affine frames, regardless of the choice of the oversampling lattice $\Lambda$.

Theorem 1.3. Suppose that $\mathcal{A}(\psi)$ and $\mathcal{A}(\phi)$ are Bessel sequences in $L^{2}\left(\mathbb{R}^{n}\right)$. Then the following are equivalent:

(i) $\mathcal{A}(\psi)$ and $\mathcal{A}(\phi)$ are dual frames,

(ii) $\mathcal{A}_{\Lambda_{0}}^{q}(\psi)$ and $\mathcal{A}_{\Lambda_{0}}^{q}(\phi)$ are dual frames for some oversampling lattice $\Lambda_{0} \subset \mathbb{Z}^{n}$,

(iii) $\mathcal{A}_{\Lambda}^{q}(\psi)$ and $\mathcal{A}_{\Lambda}^{q}(\phi)$ are dual frames for all oversampling lattices $\Lambda \subset \mathbb{Z}^{n}$.

Theorem 1.3 points at a location of the broken symmetry in the equivalence between affine and quasi-affine frames in the rational non-integer case. If such non-equivalence exists, then it can only exhibit itself for quasi-affine frames which do not have a dual quasi-affine frame. The last section of this work is devoted to showing that such phenomenon does indeed exist. For any non-integer rational 
dilation factor we give an example of a quasi-affine frame $\mathcal{A}_{\Lambda}^{q}(\psi)$ such that the corresponding affine system $\mathcal{A}(\psi)$ is not a frame.

Theorem 1.4. For each rational non-integer dilation factor $a>1$, there exists a function $\psi \in L^{2}(\mathbb{R})$ such that $\mathcal{A}_{\Lambda}^{q}(\psi)$ is a frame for any oversampling lattice $\Lambda \subset \mathbb{Z}$, but yet $\mathcal{A}(\psi)$ is not a frame.

Despite that each system $\mathcal{A}_{\Lambda}^{q}(\psi)$ is a frame, its lower frame bound drops to zero as the lattice $\Lambda$ gets more and more sparse. Hence, this example does not contradict Theorem 1.1. Moreover, in the light of Theorem 1.3 none of the quasi-affine frames $\mathcal{A}_{\Lambda}^{q}(\psi)$ can have a dual quasi-affine frame.

We end this introduction by reviewing some basic definitions. A frame sequence is a countable collection of vectors $\left\{f_{j}\right\}_{j \in \mathcal{J}}$ such that there are constants $0<C_{1} \leq$ $C_{2}<\infty$ satisfying, for all $f \in \overline{\operatorname{span}}\left\{f_{j}\right\}$,

$$
C_{1}\|f\|^{2} \leq \sum_{j \in \mathcal{J}}\left|\left\langle f, f_{j}\right\rangle\right|^{2} \leq C_{2}\|f\|^{2} .
$$

If $\overline{\operatorname{span}}\left\{f_{j}\right\}=\mathcal{H}$ for a separable Hilbert space $\mathcal{H}$, we say that the frame sequence $\left\{f_{j}\right\}_{j \in \mathcal{J}}$ is a frame for $\mathcal{H}$. If the upper bound in the above inequality holds, but not necessarily the lower bound, the sequence $\left\{f_{j}\right\}$ is said to be a Bessel sequence with Bessel constant $C_{2}$. For a Bessel sequence $\left\{f_{j}\right\}$, we define the frame operator of $\left\{f_{j}\right\}$ by

$$
S: \mathcal{H} \rightarrow \mathcal{H}, \quad S f=\sum_{j \in \mathcal{J}}\left\langle f, f_{j}\right\rangle f_{j} .
$$

If $\left\{f_{j}\right\}$ is a frame, this operator is bounded, invertible, and positive. A frame $\left\{f_{j}\right\}$ is said to be tight if we can choose $C_{1}=C_{2}$. This is equivalent to $S=C_{1} I$, where $I$ is the identity operator. If furthermore $C_{1}=C_{2}=1$, the sequence $\left\{f_{j}\right\}$ is said to be a Parseval frame.

Two Bessel sequences $\left\{f_{j}\right\}$ and $\left\{g_{j}\right\}$ are said to be dual frames if

$$
f=\sum_{j \in \mathcal{J}}\left\langle f, g_{j}\right\rangle f_{j} \quad \text { for all } f \in \mathcal{H} .
$$

It can be shown that two such Bessel sequences indeed are frames, and we shall say that the frame $\left\{g_{j}\right\}$ is dual to $\left\{f_{j}\right\}$, and vice versa. At least one dual always exists; it is given by $\left\{S^{-1} f_{j}\right\}$ and called the canonical dual.

Let $f \in L^{2}\left(\mathbb{R}^{n}\right)$ for some fixed $n \in \mathbb{N}$. The translation by $y \in \mathbb{R}^{n}$ is $T_{y} f(x)=$ $f(x-y)$; dilation by an $n \times n$ non-singular matrix $B$ is $D_{B} f(x)=|\operatorname{det} B|^{1 / 2} f(B x)$. These two operations are unitary as operators on $L^{2}\left(\mathbb{R}^{n}\right)$. Let $\Psi=\left\{\psi_{1}, \ldots, \psi_{L}\right\} \subset$ $L^{2}\left(\mathbb{R}^{n}\right)$ and let $A$ be a fixed $n \times n$ expansive matrix, i.e., all eigenvalues $\lambda$ of $A$ satisfy $|\lambda|>1$. The affine system of unitaries $\mathcal{A}$ associated with the dilation $A$ is defined as $\mathcal{A}=\left\{D_{A^{j}} T_{k}: j \in \mathbb{Z}, k \in \mathbb{Z}^{n}\right\}$, and the affine system $\mathcal{A}(\Psi)$ generated by $\Psi$ is defined as

$$
\mathcal{A}(\Psi)=\left\{\psi_{j, k}: j \in \mathbb{Z}, k \in \mathbb{Z}^{n}, \psi \in \Psi\right\},
$$

where $\psi_{j, k}=D_{A^{j}} T_{k} \psi$ for $j \in \mathbb{Z}, k \in \mathbb{Z}^{n}$. We say that $\Psi$ is a frame wavelet if $\mathcal{A}(\Psi)$ is a frame for $L^{2}\left(\mathbb{R}^{n}\right)$, and say that $\Psi$ and $\Phi$ is a pair of dual frame wavelets if their wavelet systems are dual frames. The transpose of the (fixed) dilation matrix $A$ is denoted by $B=A^{t}$. 
Following [12, the local commutant of a system of operators $\mathcal{U}$ at the point $f \in L^{2}\left(\mathbb{R}^{n}\right)$ is defined as

$$
\mathcal{C}_{f}(\mathcal{U}):=\left\{T \in B\left(L^{2}\left(\mathbb{R}^{n}\right)\right): T U f=U T f \quad \forall U \in \mathcal{U}\right\} .
$$

For $f \in L^{1}\left(\mathbb{R}^{n}\right)$, the Fourier transform is defined by

$$
\mathcal{F} f(\xi)=\hat{f}(\xi)=\int_{\mathbb{R}^{n}} f(x) \mathrm{e}^{-2 \pi i\langle\xi, x\rangle} \mathrm{d} x
$$

with the usual extension to $L^{2}\left(\mathbb{R}^{n}\right)$. We will frequently prove our results on the following subspace of $L^{2}\left(\mathbb{R}^{n}\right)$ :

$$
\mathscr{D}=\left\{f \in L^{2}\left(\mathbb{R}^{n}\right): \hat{f} \in L^{\infty}\left(\mathbb{R}^{n}\right) \text { and supp } \hat{f} \text { is compact in } \mathbb{R}^{n} \backslash\{0\}\right\},
$$

and extend the result by density arguments.

\section{Generalized Shift invariant Systems, LATtices AND OVERSAMPling}

In this section we review some fundamental properties of lattices, shift invariant systems, oversampling of shift invariant systems, mixed dual Gramians, and generalized shift invariant systems.

2.1. Lattices in $\mathbb{R}^{n}$. A lattice $\Gamma$ in $\mathbb{R}^{n}$ is a discrete subgroup under addition generated by integral linear combinations of $n$ linearly independent vectors $\left\{p_{i}\right\}_{i=1}^{n} \subset \mathbb{R}^{n}$, i.e.,

$$
\Gamma=\left\{z_{1} p_{1}+\cdots+z_{n} p_{n}: z_{1}, \ldots, z_{n} \in \mathbb{Z}\right\} .
$$

In other words, it is a set of points of the form $P \mathbb{Z}^{n}$ for a non-singular $n \times n$ matrix $P$. Let $\Gamma$ be a lattice in $\mathbb{R}^{n}$. If $\Gamma=P \mathbb{Z}^{n}$, we say that the matrix $P \in G L_{n}(\mathbb{R})$ generates the lattice $\Gamma$. A generating matrix of a given lattice is only unique up to multiplication from the right by integer matrices with determinant one in absolute value. In particular, if $\Gamma=P \mathbb{Z}^{n}$ for some $P \in G L_{n}(\mathbb{R})$, then also $\Gamma=P S \mathbb{Z}^{n}$ for any $S \in S L_{n}(\mathbb{Z})$. The determinant of $\Gamma$ is defined to be

$$
d(\Gamma)=|\operatorname{det} P|,
$$

where $P \in G L_{n}(\mathbb{R})$ is a generating matrix for $\Gamma$; note that $d(\Gamma)>0$ and $d\left(\mathbb{Z}^{n}\right)=1$. The determinant $d(\Gamma)$ is independent of the particular choice of generating matrix $P$ and equals the volume of a fundamental domain $I_{\Gamma}$ of the lattice $\Gamma$, where

$$
I_{\Gamma}=P\left([0,1)^{n}\right)=\left\{c_{1} p_{1}+\cdots+c_{n} p_{n}: 0 \leq c_{i}<1 \text { for } i=1, \ldots, n\right\}
$$

with $p_{i}$ denoting the $i$ th column of a generating matrix $P$. Note that

$$
\mathbb{R}^{n}=\bigcup_{\gamma \in \Gamma}\left(\gamma+I_{\Gamma}\right)
$$

with the union being disjoint, and that the specific shape of $I_{\Gamma}$ depends on the choice of the generating matrix $P$.

Suppose that $\Gamma \subset \Lambda$, in other words, that $\Gamma$ is a sublattice of some "denser" lattice $\Lambda$. We define the index of $\Gamma$ in $\Lambda$ as

$$
D=\frac{d(\Gamma)}{d(\Lambda)} .
$$


The index $D$ is always a positive integer. It is actually the number of copies of parallelotopes $I_{\Gamma}$ that fit inside a larger parallelotope $I_{\Lambda}$. If $D$ is the index of $\Gamma$ in $\Lambda$, we have from [6, §I.2.2],

$$
D \wedge \subset \Gamma \subset \Lambda,
$$

and, from [6, Lemma I.1],

$$
\#\{\Lambda / \Gamma\}=D \equiv d(\Gamma) / d(\Lambda),
$$

where $\#\{\Lambda / \Gamma\}$ is the order of the quotient group $\Lambda / \Gamma$. As illustrated in the following, these simple relations are often very useful. Suppose $\Gamma$ is a rational lattice, i.e., the points of the lattice have rational coordinates or, equivalently, the entries of a generating matrix $P$ are rational. In this situation we define $\tilde{\Gamma}$, the integral sublattice of $\Gamma$, by $\tilde{\Gamma}=\mathbb{Z}^{n} \cap \Gamma$, and the extended integral superlattice of $\Gamma$ by $\Gamma+\mathbb{Z}^{n}$. Using the characterization of lattices in [6. Theorem III.VI], it is straightforward to show that these point sets are actually lattices. Thus $\tilde{\Gamma}=\Gamma \cap \mathbb{Z}^{n}$ is a sublattice of $\mathbb{Z}^{n}$ with index in $\mathbb{Z}^{n}$ as

$$
D=\frac{d(\tilde{\Gamma})}{d\left(\mathbb{Z}^{n}\right)}=d(\tilde{\Gamma})
$$

and consequently,

$$
d(\tilde{\Gamma}) \mathbb{Z}^{n} \subset \tilde{\Gamma} \subset \Gamma .
$$

This shows that any rational lattice $\Gamma$ has an integral sublattice of the form $c \mathbb{Z}^{n}$, where the constant $c \in \mathbb{N}$ can be taken to be $c=d(\tilde{\Gamma})=\operatorname{vol} I_{\tilde{\Gamma}}=\#\left\{\mathbb{Z}^{n} / \tilde{\Gamma}\right\}$. Since we also have $\#\{\Gamma / \tilde{\Gamma}\}=d(\tilde{\Gamma}) / d(\Gamma)$, the above calculations show that

$$
\#\left\{\mathbb{Z}^{n} / \tilde{\Gamma}\right\}=\#\{\Gamma / \tilde{\Gamma}\} d(\Gamma) .
$$

In a similar way, we have for the extended integral superlattice of $\Gamma$

$$
\#\left\{\left(\Gamma+\mathbb{Z}^{n}\right) / \mathbb{Z}^{n}\right\}=d\left(\Gamma+\mathbb{Z}^{n}\right)^{-1}=\operatorname{vol} I_{\Gamma+\mathbb{Z}^{n}}{ }^{-1} \in \mathbb{N}
$$

and

$$
\#\left\{\left(\Gamma+\mathbb{Z}^{n}\right) / \mathbb{Z}^{n}\right\}\left(\Gamma+\mathbb{Z}^{n}\right) \subset \mathbb{Z}^{n} .
$$

The dual lattice of $\Gamma$ is given by

$$
\Gamma^{*}=\left\{\eta \in \mathbb{R}^{n}:\langle\eta, \gamma\rangle \in \mathbb{Z} \text { for } \gamma \in \Gamma\right\} ;
$$

thus if $\Gamma=P \mathbb{Z}^{n}$, then $\Gamma^{*}=\left(P^{t}\right)^{-1} \mathbb{Z}^{n}$. The determinants of dual lattices satisfy the relation

$$
d(\Gamma) d\left(\Gamma^{*}\right)=1 .
$$

If $\Gamma \subset \Lambda$, then $\Lambda^{*} \subset \Gamma^{*}$. For rational lattices $\Gamma$ and $\Lambda$ the dual lattice of $\Gamma \cap \Lambda$ and $\Gamma+\Lambda$ are $\Gamma^{*}+\Lambda^{*}$ and $\Gamma^{*} \cap \Lambda^{*}$, respectively. Dual lattices are sometimes called polar or reciprocal lattices. We refer to [6] for further basic properties of lattices.

\subsection{Shift invariant systems.}

Definition 2.1. Suppose that $\Gamma$ is a (full-rank) lattice in $\mathbb{R}^{n}$; i.e., $\Gamma=P \mathbb{Z}^{n}$ for some $n \times n$ non-singular matrix $P$. A closed subspace $W \subset L^{2}\left(\mathbb{R}^{n}\right)$ is said to be shift invariant (SI) with respect to the lattice $\Gamma$, or simply $\Gamma$-SI, if $f \in W$ implies $T_{\gamma} f \in W$ for all $\gamma \in \Gamma$. Given a countable family $\Phi \subset L^{2}\left(\mathbb{R}^{n}\right)$ and a lattice $\Gamma$, we define the $\Gamma$-SI system $E^{\Gamma}(\Phi)$ and the $\Gamma$-SI subspace $S\ulcorner(\Phi)$ by

$$
E^{\ulcorner}(\Phi)=\left\{T_{\gamma} \phi: \phi \in \Phi, \gamma \in \Gamma\right\}, \quad S^{\Gamma}(\Phi)=\overline{\operatorname{span}} E^{\Gamma}(\Phi) .
$$


We will need the following result on oversampling of shift invariant frame sequences. In case the frame sequence is actually a frame for all of $L^{2}\left(\mathbb{R}^{n}\right)$, assertion (i) below reduces to [14, Theorem 3.3]. Our proof is more elementary than [14, Theorem 3.3] and is included to illustrate how well behaved shift invariant systems are under oversampling.

Proposition 2.1. Let $\Gamma, \Gamma^{\prime}$ be lattices in $\mathbb{R}^{n}$ and $\Phi, \Psi \subset L^{2}\left(\mathbb{R}^{n}\right)$ countable sets of the same cardinality. Suppose that $\Gamma \subset \Gamma^{\prime}$ and $S^{\Gamma}(\Phi)=S^{\Gamma^{\prime}}(\Phi)$. Then the following assertions hold:

(i) If $E^{\Gamma}(\Phi)$ is a frame sequence with bounds $C_{1}, C_{2}$, then

$$
\frac{1}{\#\left\{\Gamma^{\prime} / \Gamma\right\}^{1 / 2}} E^{\Gamma^{\prime}}(\Phi)
$$

is a frame sequence with bounds $C_{1}, C_{2}$.

(ii) Suppose that $S^{\Gamma}(\Phi)=S^{\Gamma}(\Psi)=S^{\Gamma^{\prime}}(\Psi)$. If $E^{\Gamma}(\Phi)$ and $E^{\Gamma}(\Psi)$ are dual frames for $S^{\Gamma}(\Phi)$, then

$$
\frac{1}{\#\left\{\Gamma^{\prime} / \Gamma\right\}^{1 / 2}} E^{\Gamma^{\prime}}(\Phi) \quad \text { and } \quad \frac{1}{\#\left\{\Gamma^{\prime} / \Gamma\right\}^{1 / 2}} E^{\Gamma^{\prime}}(\Psi)
$$

are dual frames for $S^{\Gamma}(\Phi)$.

Proof. To prove (i) assume that there are constant $C_{1}, C_{2}>0$ such that

$$
C_{1}\|f\|^{2} \leq \sum_{\phi \in \Phi} \sum_{\gamma \in \Gamma}\left|\left\langle f, T_{\gamma} \phi\right\rangle\right|^{2} \leq C_{2}\|f\|^{2} \quad \text { for all } f \in S^{\Gamma}(\Phi) .
$$

Let $\left\{d_{1}, \ldots, d_{q}\right\}$ be a complete set of representatives of the quotient group $\Gamma^{\prime} / \Gamma$. For each $d_{r}, r=1, \ldots, q$, we then have

$$
C_{1}\|f\|^{2} \leq \sum_{\phi \in \Phi} \sum_{\gamma \in \Gamma}\left|\left\langle T_{-d_{r}} f, T_{\gamma} \phi\right\rangle\right|^{2} \leq C_{2}\|f\|^{2} \quad \text { for all } f \in S^{\Gamma}(\Phi)
$$

using the isometry of the translation operator, i.e., $\left\|T_{-d_{r}} f\right\|=\|f\|$, and the $\Gamma^{\prime}$-SI of $S^{\Gamma^{\prime}}(\Phi)=S^{\Gamma}(\Phi)$. Adding these $q$ inequalities yield

$$
q C_{1}\|f\|^{2} \leq \sum_{\phi \in \Phi} \sum_{r=1}^{q} \sum_{\gamma \in \Gamma}\left|\left\langle f, T_{d_{r}+\gamma} \phi\right\rangle\right|^{2} \leq q C_{2}\|f\|^{2},
$$

and thus,

$$
C_{1}\|f\|^{2} \leq \sum_{\phi \in \Phi} \sum_{\gamma \in \Gamma^{\prime}}\left|\left\langle f, q^{-1 / 2} T_{\gamma} \phi\right\rangle\right|^{2} \leq C_{2}\|f\|^{2} .
$$

Since $q=\#\left\{\Gamma^{\prime} / \Gamma\right\}$, assertion (i) is proved.

Let $\Phi$ and $\Psi$ be indexed by $\mathcal{I}$, i.e., $\Phi=\left\{\phi_{i}\right\}_{i \in \mathcal{I}}$ and $\Psi=\left\{\psi_{i}\right\}_{i \in \mathcal{I}}$. By our assumption we have

$$
f=\sum_{i \in \mathcal{I}} \sum_{\gamma \in \Gamma}\left\langle f, T_{\gamma} \phi_{i}\right\rangle T_{\gamma} \psi_{i} \quad \text { for all } f \in S^{\Gamma}(\Phi)=S^{\Gamma}(\Psi) ;
$$

hence, in particular,

$$
\|f\|^{2}=\sum_{i \in \mathcal{I}} \sum_{\gamma \in \Gamma}\left\langle f, T_{\gamma} \phi_{i}\right\rangle\left\langle T_{\gamma} \psi_{i}, f\right\rangle
$$


Using the same techniques as in the proof of (i) we arrive at

$$
f=\sum_{i \in \mathcal{I}} \sum_{\gamma \in \Gamma^{\prime}}\left\langle f, q^{-1 / 2} T_{\gamma} \phi_{i}\right\rangle q^{-1 / 2} T_{\gamma} \psi_{i} \quad \text { for all } f \in S^{\Gamma}(\Phi)=S^{\Gamma^{\prime}}(\Phi) \text {. }
$$

By (i) the sequences $q^{-1 / 2} E^{\Gamma^{\prime}}(\Phi)$ and $q^{-1 / 2} E^{\Gamma^{\prime}}(\Psi)$ are Bessel sequences, and (ii) is proved.

As an immediate consequence of Proposition 2.1 we have the following useful fact for SI frame sequences spanning all of $L^{2}\left(\mathbb{R}^{n}\right)$.

Corollary 2.2. Let $\Gamma$ be a lattice. If $E^{\Gamma}(\Phi)$ is a frame for $L^{2}\left(\mathbb{R}^{n}\right)$ with bounds $C_{1}, C_{2}$, then, for any superlattice $\Gamma^{\prime}$ of $\Gamma$, i.e., $\Gamma \subset \Gamma^{\prime}$,

$$
\frac{1}{\#\left\{\Gamma^{\prime} / \Gamma\right\}^{1 / 2}} E^{\Gamma^{\prime}}(\Phi)
$$

is a frame for $L^{2}\left(\mathbb{R}^{n}\right)$ with bounds $C_{1}, C_{2}$.

Corollary 2.2 is [14, Theorem 3.3] stated in terms of lattices rather than in terms of lattice generating matrices. In the matrix version the condition $\Gamma \subset \Gamma^{\prime}$ becomes the less transparent, but equivalent, condition $C^{-1} R C \in G L_{n}(\mathbb{Z})$, where $\Gamma=C \mathbb{Z}^{n}$ and $\Gamma^{\prime}=R^{-1} C \mathbb{Z}^{n}$ for $R, C \in G L_{n}(\mathbb{R})$, i.e., $E^{\Gamma}(\Phi)=\left\{T_{C k} \phi: k \in \mathbb{Z}^{n}, \phi \in \Phi\right\}$ and $E^{\Gamma^{\prime}}(\Phi)=\left\{T_{R^{-1} C k} \phi: k \in \mathbb{Z}^{n}, \phi \in \Phi\right\}$.

2.3. Oversampling SI systems. Following [3] we introduce the notion of oversampling an SI system by a rational lattice.

Definition 2.2. Let $\Gamma, \Lambda$ be rational lattices in $\mathbb{R}^{n}$, i.e., lattices with generating matrices in $G L_{n}(\mathbb{Q})$. Suppose $\Phi \subset L^{2}\left(\mathbb{R}^{n}\right)$ is a countable set. Define $O_{\Lambda}^{\Gamma}(\Phi)$, the oversampling of $E^{\Gamma}(\Phi)$ by a rational lattice $\Lambda \subset \mathbb{Q}^{n}$, as

$$
O_{\Lambda}^{\Gamma}(\Phi)=E^{\Gamma+\Lambda}\left(\frac{1}{\#\{\Lambda /(\Lambda \cap \Gamma)\}^{1 / 2}} \Phi\right) .
$$

By definition $O_{\Lambda}^{\Gamma}(\Phi)$ is always SI with respect to $\Lambda$, and if $\Lambda \subset \Gamma$, no oversampling occurs, and the oversampled system $O_{\Lambda}^{\Gamma}(\Phi)=E^{\Gamma}(\Phi)$. Moreover,

$$
\begin{aligned}
O_{\Lambda}^{\Gamma}(\Phi) & \equiv\left\{\frac{1}{\#\{\Lambda /(\Lambda \cap \Gamma)\}^{1 / 2}} T_{\omega} \phi: \phi \in \Phi, \omega \in \Gamma+\Lambda\right\} \\
& =\left\{\frac{1}{\#\{\Lambda /(\Lambda \cap \Gamma)\}^{1 / 2}} T_{d+\gamma} \phi: \phi \in \Phi, d \in[\Lambda /(\Lambda \cap \Gamma)], \gamma \in \Gamma\right\} \\
& \equiv \frac{1}{\#\{\Lambda /(\Lambda \cap \Gamma)\}^{1 / 2}} \bigcup_{d \in[\Lambda /(\Lambda \cap \Gamma)]} T_{d}\left(E^{\Gamma}(\Phi)\right),
\end{aligned}
$$

where the union runs over representatives of distinct cosets of the group $\Lambda /(\Lambda \cap \Gamma)$. Indeed, the penultimate equality is a consequence of the fact that by choosing representatives of cosets of $(\Gamma+\Lambda) / \Gamma$ in $\Lambda$, we also have representatives of $\Lambda /(\Lambda \cap \Gamma)$. Likewise, choosing the representatives of cosets of $(\Gamma+\Lambda) / \Lambda$ to be in $\Gamma$ yields representatives of $\Gamma /(\Lambda \cap \Gamma)$; hence

$$
O_{\Lambda}^{\Gamma}(\Phi)=\frac{1}{\#\{\Lambda /(\Lambda \cap \Gamma)\}^{1 / 2}} \bigcup_{d \in[\Gamma /(\Lambda \cap \Gamma)]} T_{d}\left(E^{\wedge}(\Phi)\right) .
$$


This equation also follows from the obvious symmetry in Definition 2.2 the $\Lambda$ oversampling of $E^{\Gamma}(\Phi)$ is equal to the $\Gamma$ oversampling of $E^{\wedge}(\Phi)$ up to a scaling factor. To be precise,

$$
d(\Gamma)^{1 / 2} O_{\Lambda}^{\Gamma}(\Phi)=d(\Lambda)^{1 / 2} O_{\Gamma}^{\Lambda}(\Phi),
$$

where we have used formula (2.4) to obtain the scaling factors.

2.4. Mixed dual Gramians. Let $\Lambda$ be a lattice in $\mathbb{R}^{n}$, and let $I_{\Lambda^{*}}$ denote a fundamental domain of $\Lambda^{*}$. Define the isometric, isomorphism $\mathcal{J}$ between $L^{2}\left(\mathbb{R}^{n}\right)$ and $L^{2}\left(I_{\Lambda^{*}}, \ell^{2}\left(\Lambda^{*}\right)\right)$ by

$$
\mathcal{J} f: I_{\Lambda^{*}} \rightarrow \ell^{2}\left(\Lambda^{*}\right), \quad \mathcal{J} f(\xi)=\{\hat{f}(\xi+\lambda)\}_{\lambda \in \Lambda^{*}} \quad \text { for } f \in L^{2}\left(\mathbb{R}^{n}\right) .
$$

Sequences of the form $\mathcal{J} f(\xi)$ are called fibers of $\ell^{2}\left(\Lambda^{*}\right)$ parametrized by the base space $\xi \in I_{\Lambda^{*}}$. Let $\left\{f_{i}\right\}_{i \in \mathcal{I}}$ and $\left\{g_{i}\right\}_{i \in \mathcal{I}}$ be countable collections of functions in $L^{2}\left(\mathbb{R}^{n}\right)$. By generalizing [1, Theorem 2.3], we have that $E^{\wedge}\left(\left\{f_{i}\right\}\right)$ is a frame (or Bessel sequence) in $L^{2}\left(\mathbb{R}^{n}\right)$ if, and only if, $\left\{d\left(\Lambda^{*}\right)^{1 / 2} \mathcal{J} f_{i}(\xi)\right\}_{i \in \mathcal{I}}$ is a frame (or Bessel sequence) in $\ell^{2}\left(\Lambda^{*}\right)$ for a.e. $\xi \in I_{\Lambda^{*}}$ with bounds being preserved. From this fact it is straightforward to verify that $E^{\wedge}\left(\left\{f_{i}\right\}\right)$ and $E^{\wedge}\left(\left\{g_{i}\right\}\right)$ are dual frames if, and only if, $\left\{d\left(\Lambda^{*}\right)^{1 / 2} \mathcal{J} f_{i}(\xi)\right\}_{i \in \mathcal{I}}$ and $\left\{d\left(\Lambda^{*}\right)^{1 / 2} \mathcal{J} g_{i}(\xi)\right\}_{i \in \mathcal{I}}$ are dual frames for a.e. $\xi \in I_{\Lambda^{*}}$.

Now, assume that $E^{\wedge}\left(\left\{f_{i}\right\}\right)$ and $E^{\wedge}\left(\left\{g_{i}\right\}\right)$ are Bessel sequences. For a fixed $\xi \in I_{\Lambda^{*}}$ set $t_{i}=d\left(\Lambda^{*}\right)^{1 / 2} \mathcal{J} f_{i}(\xi)$ and $u_{i}=d\left(\Lambda^{*}\right)^{1 / 2} \mathcal{J} g_{i}(\xi)$ for $i \in \mathcal{I}$. The synthesis operators for the fibers $\left\{t_{i}\right\}$ and $\left\{u_{i}\right\}$ are defined by

$$
\begin{aligned}
& T: \ell^{2}(I) \rightarrow \ell^{2}\left(\Lambda^{*}\right), \quad T\left(\left\{c_{i}\right\}\right)=\sum_{i \in \mathcal{I}} c_{i} t_{i}, \\
& U: \ell^{2}(I) \rightarrow \ell^{2}\left(\Lambda^{*}\right), \quad U\left(\left\{c_{i}\right\}\right)=\sum_{i \in \mathcal{I}} c_{i} u_{i},
\end{aligned}
$$

respectively. The analysis operators are the adjoint operators, and one finds

$$
T^{*}(a)=\left\{\left\langle a, t_{i}\right\rangle\right\}_{i \in \mathcal{I}}, \quad U^{*}(a)=\left\{\left\langle a, u_{i}\right\rangle\right\}_{i \in \mathcal{I}},
$$

for $a=\left\{a_{\lambda}\right\}_{\lambda \in \Lambda^{*}} \in \ell^{2}\left(\Lambda^{*}\right)$. The fibers $\left\{t_{i}\right\}$ and $\left\{u_{i}\right\}$ being dual frames in $\ell^{2}\left(\Lambda^{*}\right)$ means in terms of the analysis and synthesis operators that

$$
T U^{*}=I_{\ell^{2}\left(\Lambda^{*}\right)} \quad \text { or } \quad U T^{*}=I_{\ell^{2}\left(\Lambda^{*}\right)},
$$

where $I_{\ell^{2}\left(\Lambda^{*}\right)}$ is the identity operator on $\ell^{2}\left(\Lambda^{*}\right)$.

The mixed dual Gramian $\widetilde{G}=\widetilde{G}(\xi)$ is defined as $\widetilde{G}=U T^{*}$. In the standard basis $\left\{e_{k}\right\}_{k \in \Lambda^{*}}$ of $\ell^{2}\left(\Lambda^{*}\right)$ the mixed dual Gramian acts by $\left\langle\widetilde{G} e_{k}, e_{l}\right\rangle=\sum_{i \in \mathcal{I}} t_{i}(k) \overline{u_{i}(l)}$, so

$$
\widetilde{G}(\xi)=\left(d\left(\Lambda^{*}\right) \sum_{i \in \mathcal{I}} \hat{f}_{i}(\xi+k) \overline{\hat{g}_{i}(\xi+l)}\right)_{k, l \in \Lambda^{*}} .
$$

By the above, the SI systems $E^{\wedge}\left(\left\{f_{i}\right\}\right)$ and $E^{\wedge}\left(\left\{g_{i}\right\}\right)$ are dual frames if, and only if, $\widetilde{G}(\xi)=I_{\ell^{2}\left(\Lambda^{*}\right)}$ for a.e. $\xi \in I_{\Lambda^{*}}$.

The following result is a generalization of [3, Lemma 2.5]. Lemma 2.3 says that the mixed dual Gramian of a pair of oversampled SI systems is in one part simply the original mixed dual Gramian, whereas, in the other part, it has zero entries. 
Lemma 2.3. Let $\Gamma$ and $\Lambda$ be lattices, and let $\Psi=\left\{\psi_{i}\right\}_{i \in \mathcal{I}}$ and $\Phi=\left\{\phi_{i}\right\}_{i \in \mathcal{I}}$ be countable sets in $L^{2}\left(\mathbb{R}^{n}\right)$. Suppose $O_{\Lambda}^{\Gamma}(\Psi)$ and $O_{\Lambda}^{\Gamma}(\Phi)$ are Bessel sequences. Then the mixed dual Gramian of $O_{\Lambda}^{\Gamma}(\Psi)$ and $O_{\Lambda}^{\Gamma}(\Phi)$ is given for $k, l \in \Lambda^{*}$ as

$$
\widetilde{G}(\xi)_{k, l}= \begin{cases}d\left(\Gamma^{*}\right) \sum_{i \in \mathcal{I}} \hat{\psi}_{i}(\xi+k) \overline{\hat{\phi}_{i}(\xi+l)} & \text { if } k-l \in \Gamma^{*} \cap \Lambda^{*}, \\ 0 & \text { if } k-l \in \Lambda^{*} \backslash \Gamma^{*} .\end{cases}
$$

Proof. We paraphrase the oversampled systems $O_{\Lambda}^{\Gamma}(\Psi)$ and $O_{\Lambda}^{\Gamma}(\Phi)$ using (2.7), which yields

$$
O_{\Lambda}^{\Gamma}(\Psi)=E^{\wedge}\left(\Psi^{\prime}\right), \quad \text { where } \Psi^{\prime}=\bigcup_{d \in[\Gamma /(\Lambda \cap \Gamma)]}\left\{\frac{1}{\#\{\Lambda /(\Lambda \cap \Gamma)\}^{1 / 2}} T_{d} \Psi\right\},
$$

and

$$
O_{\Lambda}^{\Gamma}(\Phi)=E^{\wedge}\left(\Phi^{\prime}\right), \quad \text { where } \Phi^{\prime}=\bigcup_{d \in[\Gamma /(\Lambda \cap \Gamma)]}\left\{\frac{1}{\#\{\Lambda /(\Lambda \cap \Gamma)\}^{1 / 2}} T_{d} \Phi\right\} .
$$

Hence, by (2.9),

$$
\begin{aligned}
d\left(\Lambda^{*}\right)^{-1} \widetilde{G}(\xi)_{k, l} & =\frac{1}{\#\{\Lambda /(\Lambda \cap \Gamma)\}} \sum_{i \in \mathcal{I}} \sum_{d \in[\Gamma /(\Lambda \cap \Gamma)]}{\widehat{T_{d}}}_{i}(\xi+k){\widehat{\widehat{T_{d}}}}_{i}(\xi+l) \\
& =\frac{1}{\#\{\Lambda /(\Lambda \cap \Gamma)\}}\left(\sum_{d \in[\Gamma /(\Lambda \cap \Gamma)]} \mathrm{e}^{-2 \pi i\langle k-l, d\rangle}\right) \sum_{i \in \mathcal{I}} \hat{\psi}_{i}(\xi+k) \overline{\hat{\phi}_{i}(\xi+l)} .
\end{aligned}
$$

Using Lemma 3.6 and $\#\{\Gamma /(\Lambda \cap \Gamma)\} / \#\{\Lambda /(\Lambda \cap \Gamma)\}=d(\Lambda) / d(\Gamma)=d\left(\Gamma^{*}\right) / d\left(\Lambda^{*}\right)$ yields (2.10).

2.5. Generalized shift invariant systems. Generalized shift invariant systems were introduced and studied in the work of Hernández, Labate, and Wilson [13, and independently by Ron and Shen $[23]$.

Definition 2.3. For a collection of functions $\left\{g_{p}\right\}_{p \in \mathcal{P}}$, a generalized shift invariant (GSI) system is defined as

$$
\bigcup_{p \in \mathcal{P}} E^{\Gamma_{p}}\left(g_{p}\right)
$$

where $\left\{\Gamma_{p}\right\}_{p \in \mathcal{P}}$ is a countable collection of lattices in $\mathbb{R}^{n}$. The $\Gamma_{p}$-SI system $E^{\Gamma_{p}}\left(g_{p}\right)$ is said to be the pth layer of the GSI system.

Letting $\Phi=\left\{g_{p}\right\}_{p \in \mathcal{P}}$ and $\Gamma=\Gamma_{p}$ for each $p \in \mathcal{P}$ in (2.11) for a GSI system, we recover the SI system $E^{\ulcorner}(\Phi)$. Moreover, a GSI system is SI if there exists a (sparse) lattice $\Gamma$ so that $\Gamma \subset \Gamma_{p}$ for each $p \in \mathcal{P}$. Furthermore, if $C_{p} \in G L_{n}(\mathbb{R})$ is chosen such that $\Gamma_{p}=C_{p} \mathbb{Z}^{n}$ for each $p \in \mathcal{P}$, then the GSI system in (2.11) takes the form

$$
\left\{T_{C_{p} k} g_{p}: k \in \mathbb{Z}^{n}, p \in \mathcal{P}\right\} .
$$

We will use the following results about GSI systems from [13. Here, we state the results from [13] in terms of lattices in $\mathbb{R}^{n}$ rather than in terms of (2.12) and matrices $\left\{C_{p}\right\}$. The reason behind this convention is that a matrix $C_{p}$ satisfying $\Gamma_{p}=C_{p} \mathbb{Z}^{n}$ is not unique and most of our conditions simplify when stated in terms of lattices rather than matrices. 
Theorem 2.4 (Theorem 2.1 in [13]). Let $\mathcal{P}$ be a countable set, $\left\{g_{p}\right\}_{p \in \mathcal{P}}$ a collection of functions in $L^{2}\left(\mathbb{R}^{n}\right)$ and $\left\{\Gamma_{p}\right\}_{p \in \mathcal{P}}$ a collection of lattices in $\mathbb{R}^{n}$. Assume the local integrability condition (LIC):

$$
L(f):=\sum_{p \in \mathcal{P}} \sum_{m \in \Gamma_{p}^{*}} \int_{\operatorname{supp} \hat{f}}|\hat{f}(\xi+m)|^{2} d\left(\Gamma_{p}^{*}\right)\left|\hat{g}_{p}(\xi)\right|^{2} \mathrm{~d} \xi<\infty \quad \text { for all } f \in \mathscr{D} .
$$

Then the GSI system $\bigcup_{p \in \mathcal{P}} E^{\Gamma_{p}}\left(g_{p}\right)$ is a Parseval frame for $L^{2}\left(\mathbb{R}^{n}\right)$ if, and only if,

$$
\sum_{p \in \mathcal{P}} d\left(\Gamma_{p}^{*}\right) \hat{g}_{p}(\xi) \overline{\hat{g}_{p}(\xi+\alpha)}=\delta_{\alpha, 0} \quad \text { for a.e. } \xi \in \mathbb{R}^{n}
$$

for each $\alpha \in \bigcup_{p \in \mathcal{P}} \Gamma_{p}^{*}$.

The fact that LIC, in general, is necessary can be found in [4, Example 3.2]. We also recall the relation between the determinants of dual lattices $d\left(\Gamma_{p}^{*}\right)=1 / d\left(\Gamma_{p}\right)$.

Proposition 2.5 (Proposition 2.4 in [13]). Let $\mathcal{P}$ be a countable set, $\left\{g_{p}\right\}_{p \in \mathcal{P}}$ a collection of functions in $L^{2}\left(\mathbb{R}^{n}\right)$ and $\left\{\Gamma_{p}\right\}_{p \in \mathcal{P}}$ a collection of lattices in $\mathbb{R}^{n}$. Assume that the LIC given by (2.13) holds. Then, for each $f \in \mathscr{D}$, the function

$$
w(x)=\sum_{p \in \mathcal{P}} \sum_{k \in \Gamma_{p}}\left|\left\langle T_{x} f, T_{k} g_{p}\right\rangle\right|^{2}
$$

is a continuous function that coincides pointwise with the absolutely convergent series

$$
w(x)=\sum_{p \in \mathcal{P}} \sum_{m \in \Gamma_{p}^{*}} \hat{w}_{p}(m) \mathrm{e}^{2 \pi i\langle m, x\rangle}
$$

where

$$
\hat{w}_{p}(m)=d\left(\Gamma_{p}^{*}\right) \int_{\mathbb{R}^{n}} \hat{f}(\xi) \overline{\hat{f}(\xi+m)} \overline{\hat{g}_{p}(\xi)} \hat{g}_{p}(\xi+m) \mathrm{d} \xi .
$$

The function $w$ in (2.16) is an almost periodic function. In case the GSI system from Proposition 2.5 is a $\Gamma$-SI system for some lattice $\Gamma$, the function $w$ is actually $\Gamma$-periodic and can thus be considered as a regular Fourier series on the fundamental parallelotope $I_{\Gamma}$.

Proposition 2.6 (Proposition 4.1 in [13]). Let $\mathcal{P}$ be a countable set, $\left\{g_{p}\right\}_{p \in \mathcal{P}}$ a collection of functions in $L^{2}\left(\mathbb{R}^{n}\right)$ and $\left\{\Gamma_{p}\right\}_{p \in \mathcal{P}}$ a collection of lattices in $\mathbb{R}^{n}$. If the GSI system $\bigcup_{p \in \mathcal{P}} E^{\Gamma_{p}}\left(g_{p}\right)$ is a Bessel sequence with bound $C_{2}>0$, then

$$
\sum_{p \in \mathcal{P}}\left|\hat{g}_{p}(\xi)\right|^{2} / d\left(\Gamma_{p}\right) \leq C_{2} \quad \text { for a.e. } \xi \in \mathbb{R}^{n} .
$$

The following result is a generalization of Proposition 5.6 in [13]. The result states that the local integrability condition for affine systems $\mathcal{A}(\psi)$ is equivalent to local integrability of a Calderón sum (2.19); hence the name of the condition.

Proposition 2.7. Let $A \in G L_{n}(\mathbb{R})$ be expansive and $\psi \in L^{2}\left(\mathbb{R}^{n}\right)$. Then

$$
\sum_{j \in \mathbb{Z}}\left|\hat{\psi}\left(B^{-j} \xi\right)\right|^{2} \in L_{\mathrm{loc}}^{1}\left(\mathbb{R}^{n} \backslash\{0\}\right)
$$


if, and only if,

$$
\begin{aligned}
L(f) & =\sum_{j \in \mathbb{Z}} \sum_{m \in \mathbb{Z}^{n}} \int_{\operatorname{supp} \hat{f}}\left|\hat{f}\left(\xi+B^{j} m\right)\right|^{2}\left|\operatorname{det} A^{j}\right|\left|\mathcal{F} D_{A^{j}} \psi(\xi)\right|^{2} \mathrm{~d} \xi \\
& =\sum_{j \in \mathbb{Z}} \sum_{m \in \mathbb{Z}^{n}} \int_{\operatorname{supp} \hat{f}}\left|\hat{f}\left(\xi+B^{j} m\right)\right|^{2}\left|\hat{\psi}\left(B^{-j} \xi\right)\right|^{2} \mathrm{~d} \xi<\infty \quad \text { for all } f \in \mathcal{D} .
\end{aligned}
$$

In the proof of Proposition 2.7 we use the following elementary lattice counting lemma.

Lemma 2.8. Let $B \in G L_{n}(\mathbb{R})$ be expansive and $R>0$. Then there exists $C>0$ such that

(2.21) $\#\left\{\left(\xi+B^{j} \mathbb{Z}^{n}\right) \cap \mathbf{B}(0, R)\right\} \leq C \max \left(1,|\operatorname{det} B|^{-j}\right) \quad$ for any $j \in \mathbb{Z}, \xi \in \mathbb{R}^{n}$.

Proof. Since the matrix $B$ is expansive, there exists $J \in \mathbb{Z}$ such that

$$
\mathbf{B}(0, \sqrt{n}) \subset B^{-j}(\mathbf{B}(0, R)) \quad \text { for all } j \leq J .
$$

For the same reason, once $J$ is fixed, there exists $R_{0}>0$ such that

$$
B^{-j}(\mathbf{B}(0, R)) \subset \mathbf{B}\left(0, R_{0}\right) \quad \text { for all } j>J .
$$

Let

$$
K_{j}=\left\{k \in \mathbb{Z}^{n}: \xi+B^{j} k \in \mathbf{B}(0, R)\right\}=\left\{k \in \mathbb{Z}^{n}: B^{-j} \xi+k \in B^{-j}(\mathbf{B}(0, R))\right\} .
$$

Using (2.22) and (2.23),

$$
\bigcup_{k \in K_{j}}\left(B^{-j} \xi+k+[0,1]^{n}\right) \subset B^{-j}(\mathbf{B}(0, R))+\mathbf{B}(0, \sqrt{n}) \subset \begin{cases}2 B^{-j}(\mathbf{B}(0, R)) & \text { for } j \leq J, \\ \mathbf{B}\left(0, R_{0}+\sqrt{n}\right) & \text { for } j>J .\end{cases}
$$

Thus,

$$
\# K_{j}=\left|\bigcup_{k \in K_{j}}\left(B^{-j} \xi+k+[0,1]^{n}\right)\right| \leq \begin{cases}c_{n}(2 R)^{n}|\operatorname{det} B|^{-j} & \text { for } j \leq J \\ c_{n}\left(R_{0}+\sqrt{n}\right)^{n} & \text { for } j>J\end{cases}
$$

where $c_{n}=|\mathbf{B}(0,1)|$. This immediately implies (2.21).

Proof of Proposition 2.7. Assume (2.19). Let $f \in \mathscr{D}$ and choose $R>1$ such that

$$
\operatorname{supp} \hat{f} \subset\left\{\xi \in \mathbb{R}^{n}: \frac{1}{R}<|\xi|<R\right\} .
$$

Since the matrix $B$ is expansive, there exists a constant $K \in \mathbb{N}$ such that each trajectory $\left\{B^{j} \xi\right\}_{j \in \mathbb{Z}}$ hits the above annulus at most $K$ times. Thus,

$$
\#\left\{j \in \mathbb{Z}: \xi \in B^{-j}(\operatorname{supp} \hat{f})\right\} \leq K .
$$

On the other hand, by Lemma 2.8 we have that, for any $\xi \in \mathbb{R}^{n}$,

$$
\#\left\{\left(\xi+B^{j} \mathbb{Z}^{n}\right) \cap \operatorname{supp} \hat{f}\right\} \leq C \max \left(1,|\operatorname{det} B|^{-j}\right) .
$$


Combining the last two estimates,

$$
\begin{aligned}
L(f) & \leq \sum_{j \in \mathbb{Z}}\|\hat{f}\|_{\infty}^{2} C \max \left(1,|\operatorname{det} B|^{-j}\right) \int_{\operatorname{supp} \hat{f}}\left|\hat{\psi}\left(B^{-j} \xi\right)\right|^{2} \mathrm{~d} \xi \\
& \leq\|\hat{f}\|_{\infty}^{2} C \sum_{j \geq 0} \int_{\operatorname{supp} \hat{f}}\left|\hat{\psi}\left(B^{-j} \xi\right)\right|^{2} \mathrm{~d} \xi+\|\hat{f}\|_{\infty}^{2} C \sum_{j<0} \int_{B^{-j}(\operatorname{supp} \hat{f})}|\hat{\psi}(\xi)|^{2} \mathrm{~d} \xi \\
& \leq\|\hat{f}\|_{\infty}^{2} C \int_{\operatorname{supp} \hat{f}} \sum_{j \geq 0}\left|\hat{\psi}\left(B^{-j} \xi\right)\right|^{2} \mathrm{~d} \xi+\|\hat{f}\|_{\infty}^{2} C K \int_{\mathbb{R}^{n}}|\hat{\psi}(\xi)|^{2} \mathrm{~d} \xi<\infty .
\end{aligned}
$$

The last inequality is a consequence of (2.19) and $\psi \in L^{2}\left(\mathbb{R}^{n}\right)$.

Conversely, if $L(f)<\infty$ for all $f \in \mathscr{D}$, then in particular by choosing $\hat{f}=\chi_{E}$ for a compact set $E \subset \mathbb{R}^{n} \backslash\{0\}$ we have

$$
\int_{E} \sum_{j \in \mathbb{Z}}\left|\hat{\psi}\left(B^{-j} \xi\right)\right|^{2} \mathrm{~d} \xi=\sum_{j \in \mathbb{Z}} \int_{E}\left|\hat{\psi}\left(B^{-j} \xi\right)\right|^{2} \mathrm{~d} \xi \leq L(f)<\infty .
$$

Since the set $E$ was arbitrarily chosen, the validity of (2.19) follows.

Remark 1. One should add that (2.19) and thus (2.20) hold if, and only if, the Bessel-like condition holds on the dense subspace $\mathscr{D}$ :

$$
\sum_{j \in \mathbb{Z}} \sum_{k \in \mathbb{Z}^{n}}\left|\left\langle f, \psi_{j, k}\right\rangle\right|^{2}<\infty \quad \text { for all } f \in \mathscr{D} .
$$

Indeed, this fact is a consequence of [2, Lemma 3.1], which holds for real expansive dilations.

\section{Oversampling AFFine SYSTEMS Into QUASI-AFFInE SYSTEMS}

In this section we show that the frame property is preserved when going from affine to quasi-affine systems. To characterize under what conditions we can also go from quasi-affine to affine systems, we introduce a new family of oversampled quasi-affine systems. We then show that an affine system is a frame if, and only if, the corresponding family of quasi-affine systems are frames with uniform frame bounds.

3.1. Properties of quasi-affine systems. For a rational lattice $\Lambda$ we introduce the notion of a $\Lambda$-oversampled quasi-affine system.

Definition 3.1. Let $A \in G L_{n}(\mathbb{Q})$ be a rational, expansive matrix, and let $\Lambda$ be rational lattice in $\mathbb{R}^{n}$, i.e., $\Lambda=P \mathbb{Z}^{n}$ with $P \in G L_{n}(\mathbb{Q})$. Suppose $\Psi \subset L^{2}\left(\mathbb{R}^{n}\right)$ is a finite set. Define $\mathcal{A}_{\Lambda}^{q}(\Psi)$ the $\Lambda$-oversampled quasi-affine system by

$$
\mathcal{A}_{\Lambda}^{q}(\Psi)=\bigcup_{j \in \mathbb{Z}} O_{\Lambda}^{A^{-j} \mathbb{Z}^{n}}\left(D_{A^{j}} \Psi\right)
$$

When $\Lambda=\mathbb{Z}^{n}$ we often drop the subscript $\Lambda$, and we say that $\mathcal{A}^{q}(\Psi)=\mathcal{A}_{\mathbb{Z}^{n}}^{q}(\Psi)$ is the standard quasi-affine system.

By definition $\mathcal{A}_{\Lambda}^{q}(\Psi)$ is SI with respect to $\Lambda$. Note that we need to assume that the dilation $A$ and the lattice $\Lambda$ are rational in order to guarantee lattice structure of $A^{-j} \mathbb{Z}^{n}+\Lambda$ for each $j \in \mathbb{Z}$. If $\Lambda=\mathbb{Z}^{n}$, we recover the usual quasi-affine system, i.e., $\mathcal{A}_{\Lambda}^{q}(\Psi)=\mathcal{A}^{q}(\Psi)$, introduced in [3]. 
We will use the following notation throughout this paper. The translation lattice for the affine system at scale $j \in \mathbb{Z}$ is denoted by $\Gamma_{j}=A^{-j} \mathbb{Z}^{n}$; its $\Lambda$-sublattice is $\widetilde{\Gamma}_{j}=A^{-j} \mathbb{Z}^{n} \cap \Lambda$ and its $\Lambda$-extended superlattice is $\mathrm{K}_{j}=A^{-j} \mathbb{Z}^{n}+\Lambda$. Note that $\mathrm{K}_{j}$ is the translation lattice for the $\Lambda$-oversampled quasi-affine system at scale $j \in \mathbb{Z}$. Finally, for $J \in \mathbb{N}$, let

$$
\mathrm{M}_{J}=\bigcap_{|j| \leq J} \Gamma_{j} \equiv \bigcap_{|j| \leq J} A^{j} \mathbb{Z}^{n}
$$

and note that $M_{J}$ is an integral lattice. Summarizing, we will use the following lattices, together with their dual lattices:

$$
\begin{aligned}
\Gamma_{j} & =A^{-j} \mathbb{Z}^{n}, & \Gamma_{j}^{*} & =B^{j} \mathbb{Z}^{n}, \\
\widetilde{\Gamma}_{j} & =A^{-j} \mathbb{Z}^{n} \cap \Lambda, & \widetilde{\Gamma}_{j}^{*} & =B^{j} \mathbb{Z}^{n}+\Lambda^{*}, \\
\mathrm{~K}_{j} & =A^{-j} \mathbb{Z}^{n}+\Lambda, & \mathrm{K}_{j}^{*} & =B^{j} \mathbb{Z}^{n} \cap \Lambda^{*}, \\
\mathrm{M}_{J} & =\bigcap_{|j| \leq J} A^{j} \mathbb{Z}^{n}, & \mathrm{M}_{J}^{*} & =\underset{|j| \leq J}{+} B^{j} \mathbb{Z}^{n}=B^{-J} \mathbb{Z}^{n}+\cdots+B^{J} \mathbb{Z}^{n} .
\end{aligned}
$$

Let $\Psi, \Phi \subset L^{2}\left(\mathbb{R}^{n}\right)$ be finite sets. For $j \in \mathbb{Z}$ and $f \in L^{2}\left(\mathbb{R}^{n}\right)$ define the affine functionals

$$
K_{j}(f)=\sum_{g \in E^{A^{-j} \mathbb{Z}^{n}\left(D_{A^{j}} \Psi\right)}}|\langle f, g\rangle|^{2}, \quad N(f, \Psi)=\sum_{j \in \mathbb{Z}} K_{j}(f)=\sum_{g \in \mathcal{A}(\Psi)}|\langle f, g\rangle|^{2}
$$

and quasi-affine functionals

$$
K_{\Lambda, j}^{q}(f)=\sum_{g \in O_{\Lambda}^{A-j} \mathbb{Z}^{n}\left(D_{A^{j}} \Psi\right)}|\langle f, g\rangle|^{2}, \quad N_{\Lambda}^{q}(f, \Psi)=\sum_{j \in \mathbb{Z}} K_{\Lambda, j}^{q}(f)=\sum_{g \in \mathcal{A}_{\Lambda}^{q}(\Psi)}|\langle f, g\rangle|^{2} .
$$

Whenever unambiguous, we drop the reference to the set of generators and simply write $N(f)$ and $N_{\Lambda}^{q}(f)$.

Before going deeper into our investigation we illustrate the notion of a quasiaffine system in a few specific situations.

Example 1. Let $J \in \mathbb{N}$ and consider the quasi-affine system obtained by oversampling with respect to $\mathrm{M}_{J}=\bigcap_{|j| \leq J} A^{j} \mathbb{Z}^{n}$ introduced above. Since $A^{-j} \mathbb{Z}^{n}+\mathrm{M}_{J}=$ $A^{-j} \mathbb{Z}^{n}$ and $A^{-j} \mathbb{Z}^{n} \cap \mathrm{M}_{J}=\mathrm{M}_{J}$ for $|j| \leq J$, we see that

$$
O_{\mathrm{M}_{J}}^{A^{-j} \mathbb{Z}^{n}}\left(D_{A^{j}} \Psi\right)=E^{A^{-j} \mathbb{Z}^{n}+\mathrm{M}_{J}}\left(\#\left\{\mathrm{M}_{J} / \mathrm{M}_{J}\right\}^{-1 / 2} D_{A^{j}} \Psi\right)=E^{A^{-j} \mathbb{Z}^{n}}\left(D_{A^{j}} \Psi\right),
$$

for $|j| \leq J$. Hence with this oversampling lattice, the scales $|j| \leq J$ for the affine system

$$
\mathcal{A}(\Psi)=\bigcup_{j \in \mathbb{Z}} E^{A^{-j} \mathbb{Z}^{n}}\left(D_{A^{j}} \Psi\right)
$$

and the $\mathrm{M}_{J}$-oversampled quasi-affine system

$$
\mathcal{A}_{\mathrm{M}_{J}}^{q}(\Psi)=\bigcup_{j \in \mathbb{Z}} O_{\mathrm{M}_{J}}^{A^{-j} \mathbb{Z}^{n}}\left(D_{A^{j}} \Psi\right)
$$

coincide. 
Example 2. Suppose $A \in G L_{n}(\mathbb{Z})$ is integer valued. Let $\Lambda=A^{l} \mathbb{Z}^{n}$ for some $l \in \mathbb{Z}$. Then the $\Lambda$-oversampled quasi-affine system is just a dilated version of standard quasi-affine system (1.2). To be precise, we have the following relation:

$$
\mathcal{A}_{A^{l} \mathbb{Z}^{n}}^{q}(\Psi)=D_{A^{-l}}\left(\mathcal{A}^{q}(\Psi)\right) \text {. }
$$

To see this note that

$$
A^{-j} \mathbb{Z}^{n}+\Lambda=A^{-j} \mathbb{Z}^{n}+A^{l} \mathbb{Z}^{n}= \begin{cases}A^{l} \mathbb{Z}^{n}, & j<-l, \\ A^{-j} \mathbb{Z}^{n}, & j \geq-l,\end{cases}
$$

and that

$\#\left\{A^{l} \mathbb{Z}^{n} /\left(A^{l} \mathbb{Z}^{n} \cap A^{-j} \mathbb{Z}^{n}\right)\right\}= \begin{cases}\#\left\{A^{l} \mathbb{Z}^{n} / A^{-j} \mathbb{Z}^{n}\right\}=\frac{d\left(\mathbb{Z}^{n}\right)}{d\left(A^{j+l} \mathbb{Z}^{n}\right)}=\frac{1}{\left|\operatorname{det} A^{j+l}\right|}, & j<-l, \\ \#\left\{A^{l} \mathbb{Z}^{n} / A^{l} \mathbb{Z}^{n}\right\}=1, & j \geq-l,\end{cases}$

whereby we have

$$
\mathcal{A}_{A^{l} \mathbb{Z}^{n}}^{q}(\Psi)=\bigcup_{j \geq-l} E^{A^{-j} \mathbb{Z}^{n}}\left(D_{A^{j}} \Psi\right) \cup \bigcup_{j<-l} E^{A^{l} \mathbb{Z}^{n}}\left(|\operatorname{det} A|^{(j+l) / 2} D_{A^{j}} \Psi\right) .
$$

Recall that

$$
\mathcal{A}^{q}(\Psi)=\bigcup_{j \geq 0} E^{A^{-j} \mathbb{Z}^{n}}\left(D_{A^{j}} \Psi\right) \cup \bigcup_{j<0} E^{\mathbb{Z}^{n}}\left(|\operatorname{det} A|^{j / 2} D_{A^{j}} \Psi\right),
$$

and the validity of (3.8) follows by $D_{A^{-l}} T_{k}=T_{A^{l} k} D_{A^{-l}}$ and a change of variables.

Example 3. The quasi-affine system has a relatively simple algebraic form in one dimension. Suppose $a=p / q \in \mathbb{Q}$ is a dilation factor, where $|a|>1$ and $p, q \in \mathbb{Z}$ are relatively prime. Let $\Lambda \subset \mathbb{Z}$ be a lattice. For simplicity, we assume that $\Lambda=p^{J_{1}} q^{J_{2}} r \mathbb{Z}$ for some $J_{1}, J_{2} \in \mathbb{N}_{0}, r \in \mathbb{N}$, where $p q$ and $r$ are relatively prime. Then, the quasi-affine system $\mathcal{A}_{\Lambda}^{q}(\Psi)$ associated with $a$ is given by

$$
\mathcal{A}_{\Lambda}^{q}(\Psi)=\left\{\tilde{\psi}_{j, k}: j, k \in \mathbb{Z}, \psi \in \Psi\right\} .
$$

Here, for $\psi \in L^{2}(\mathbb{R})$ and $j, k \in \mathbb{Z}$, we set

$$
\tilde{\psi}_{j, k}(x)= \begin{cases}|a|^{j / 2}|q|^{\left(J_{2}-j\right) / 2} \psi\left(a^{j} x-q^{J_{2}-j} k\right) & \text { if } j>J_{2}, \\ |a|^{j / 2} \psi\left(a^{j} x-k\right) & \text { if }-J_{1} \leq j \leq J_{2}, \\ |a|^{j / 2}|p|^{\left(j+J_{1}\right) / 2} \psi\left(a^{j} x-p^{j+J_{1}} k\right) & \text { if } j<-J_{1} .\end{cases}
$$

Note that the above convention for $\tilde{\psi}_{j, k}$ in the case when $\Lambda=\mathbb{Z}$ becomes the rationally dilated quasi-affine system (1.3) introduced by the first author in [3]. In particular, if the dilation factor $a$ is an integer, this is the original quasi-affine system of Ron and Shen [20]. To show (3.9) note that

$$
\begin{array}{rl}
a^{-j} & \mathbb{Z}=a^{-j} \mathbb{Z}+p^{J_{1}} q^{J_{2}} r \mathbb{Z} \\
& = \begin{cases}p^{-j}\left(q^{j} \mathbb{Z}+p^{J_{1}+j} q^{J_{2}} r \mathbb{Z}\right)=p^{-j} q^{\min \left(j, J_{2}\right)} \mathbb{Z} & \text { for } j \geq 0, \\
q^{j}\left(p^{-j} \mathbb{Z}+p^{J_{1}} q^{J_{2}-j} r \mathbb{Z}\right)=q^{j} p^{\min \left(-j, J_{1}\right)} \mathbb{Z} & \text { for } j<0,\end{cases} \\
& = \begin{cases}p^{-j} q^{J_{2}} \mathbb{Z} & \text { for } j>J_{2}, \\
a^{-j} \mathbb{Z} & \text { for }-J_{1} \leq j \leq J_{2}, \\
p^{J_{1}} q^{j} \mathbb{Z} & \text { for } j<-J_{1} .\end{cases}
\end{array}
$$

Hence, one needs to oversample at a rate $|q|^{j-J_{2}}$ if $j>J_{2}$ (or $|p|^{-J_{1}-j}$ if $j<-J_{1}$ ) to obtain the quasi-affine system $\mathcal{A}_{\Lambda}^{q}(\Psi)$ from the affine system $\mathcal{A}(\Psi)$. Note that 
in the intermediate range $-J_{1} \leq j \leq J_{2}$, no oversampling is required and both systems coincide at these scales. Also note that the choice $J_{1}=J_{2}$ corresponds to oversampling by $\mathrm{M}_{J_{1}}$; see Example 1

Remark 2 . Let $\Lambda$ be a rational lattice, and consider the $\Lambda$-oversampled quasi-affine system $\mathcal{A}_{\Lambda}^{q}(\Psi)$. By definition this system is $\Lambda$-SI. Take a rational superlattice $\Lambda^{\prime}$ of $\Lambda$, i.e., $\Lambda \subset \Lambda^{\prime}$. Then the further oversampled system $\mathcal{A}_{\Lambda^{\prime}}^{q}(\Psi)$ is obviously $\Lambda^{\prime}$-SI; moreover, it can be written in terms of $\mathcal{A}_{\Lambda}^{q}(\Psi)$ as

$$
\mathcal{A}_{\Lambda^{\prime}}^{q}(\Psi)=\frac{1}{\#\left\{\Lambda^{\prime} / \Lambda\right\}^{1 / 2}} \bigcup_{d \in\left[\Lambda^{\prime} / \Lambda\right]} T_{d}\left(\mathcal{A}_{\Lambda}^{q}(\Psi)\right) .
$$

By Corollary 2.2 we have the following useful result for oversampled quasi-affine frames:

Lemma 3.1. Let $A \in G L_{n}(\mathbb{Q})$. Suppose $\Lambda \subset \Lambda^{\prime}$ for rational lattices $\Lambda, \Lambda^{\prime}$. Then if $\mathcal{A}_{\Lambda}^{q}(\Psi)$ is a frame for $L^{2}\left(\mathbb{R}^{n}\right)$ with bounds $C_{1}, C_{2}, \mathcal{A}_{\Lambda^{\prime}}^{q}(\Psi)$ is a frame for $L^{2}\left(\mathbb{R}^{n}\right)$ with bounds $C_{1}, C_{2}$.

3.2. Affine and quasi-affine systems as GSI systems. Since affine and quasiaffine systems are GSI systems, the results from Section 2.5 can be applied to these systems; see [13, 14]. We restate some of these results in terms of lattices in $\mathbb{R}^{n}$. The quasi-affine system $\mathcal{A}_{\Lambda}^{q}(\Psi)$ introduced above can be expressed as a GSI system (2.11) by taking $\mathcal{P}=\{(j, l): j \in \mathbb{Z}, l=1, \ldots, L\}$ and

$$
\begin{aligned}
\Gamma_{p} & =\Gamma_{(j, l)}=A^{-j} \mathbb{Z}^{n}+\Lambda, \\
g_{p}(x)=g_{(j, l)}(x) & =\#\left\{\Lambda /\left(\Lambda \cap A^{-j} \mathbb{Z}^{n}\right)\right\}^{-1 / 2} D_{A^{j}} \psi_{l}(x)
\end{aligned}
$$

for all $p \in \mathcal{P}$.

By applying Proposition 2.6 to affine and quasi-affine systems we immediately have the following result; see also [3, Proposition 4.5].

Proposition 3.2. Suppose that $\Psi \subset L^{2}\left(\mathbb{R}^{n}\right)$ and that either of the following holds:

(a) $A \in G L_{n}(\mathbb{R})$ is expansive and $\mathcal{A}(\Psi)$ is a Bessel sequence with bound $C_{2}$,

(b) $A \in G L_{n}(\mathbb{Q})$ is expansive and $\mathcal{A}_{\Lambda}^{q}(\Psi)$ is a Bessel sequence with bound $C_{2}$ for some rational lattice $\Lambda$.

Then

$$
\sum_{\psi \in \Psi} \sum_{j \in \mathbb{Z}}\left|\hat{\psi}\left(B^{j} \xi\right)\right|^{2} \leq C_{2} \quad \text { for a.e. } \xi \in \mathbb{R}^{n} .
$$

For the $\Lambda$-oversampled quasi-affine systems we have the following result on the quasi-affine functional $w_{\Lambda}^{q}$ defined below.

Proposition 3.3. Let $A \in G L_{n}(\mathbb{Q})$ be expansive, $\Psi=\left\{\psi_{1}, \ldots, \psi_{L}\right\} \subset L^{2}\left(\mathbb{R}^{n}\right)$, and let $\Lambda$ be a rational lattice. Suppose that each $\psi \in \Psi$ satisfies condition (2.19). Then, for each $f \in \mathscr{D}$, the $\Lambda$-periodic function

$$
w_{\Lambda}^{q}(x)=\sum_{g \in \mathcal{A}_{\Lambda}^{q}(\Psi)}\left|\left\langle T_{x} f, g\right\rangle\right|^{2}=\sum_{l=1}^{L} \sum_{j \in \mathbb{Z}} \sum_{k \in \mathrm{K}_{j}}\left|\left\langle T_{x} f, d_{j} T_{k} D_{A^{j}} \psi_{l}\right\rangle\right|^{2},
$$


where $d_{j}=\#\left\{\Lambda /\left(\Lambda \cap A^{-j} \mathbb{Z}^{n}\right)\right\}^{-1 / 2}$ and $\mathrm{K}_{j}$ is given by (3.3) , is a continuous function that coincides pointwise with the $(\Lambda$-periodic) absolutely convergent series

$$
w_{\Lambda}^{q}(x)=\sum_{l=1}^{L} \sum_{j \in \mathbb{Z}} \sum_{\mu \in \mathrm{K}_{j}^{*}} b_{j, l}(\mu) \mathrm{e}^{2 \pi i\langle\mu, x\rangle},
$$

where

$$
b_{j, l}(\mu)=\int_{\mathbb{R}^{n}} \hat{f}(\xi) \overline{\hat{f}(\xi+\mu)} \overline{\hat{\psi}_{l}\left(B^{-j} \xi\right)} \hat{\psi}_{l}\left(B^{-j}(\xi+\mu)\right) \mathrm{d} \xi .
$$

Proof. The result follows by an application of Proposition 2.5 to quasi-affine systems. In order to apply Proposition 2.5 we need to verify the LIC condition (2.13) for quasi-affine systems, i.e., that

$$
L_{\Lambda}^{q}(f):=\sum_{l=1}^{L} \sum_{j \in \mathbb{Z}} \sum_{\mu \in \mathrm{K}_{j}^{*}} \int_{\operatorname{supp} \hat{f}}|\hat{f}(\xi+\mu)|^{2}\left|\hat{\psi}_{l}\left(B^{-j} \xi\right)\right|^{2} \mathrm{~d} \xi<\infty
$$

holds for $f \in \mathcal{D}$. Since each $\psi \in \Psi$ satisfies condition (2.19), Proposition 2.7 tells us that the LIC condition for affine systems is satisfied, i.e., that $L(f)<\infty$. Finally, the estimate in (3.16) follows by

$$
L_{\Lambda}^{q}(f) \leq \sum_{l=1}^{L} \sum_{j \in \mathbb{Z}} \sum_{m \in \mathbb{Z}^{n}} \int_{\operatorname{supp} \hat{f}}\left|\hat{f}\left(\xi+B^{j} m\right)\right|^{2}\left|\hat{\psi}_{l}\left(B^{-j} \xi\right)\right|^{2} \mathrm{~d} \xi \equiv L(f)<\infty,
$$

where we have used the fact that $\mathrm{K}_{j}^{*} \subset B^{j} \mathbb{Z}^{n}$ for all $j \in \mathbb{Z}$. Consequently, the expression in (3.15) follows directly from (2.17) by

$$
1 / d\left(\mathrm{~K}_{j}^{*}\right)=d\left(\mathrm{~K}_{j}\right)=\frac{\left|\operatorname{det} A^{-j}\right|}{\#\left\{\Lambda /\left(\Lambda \cap A^{-j} \mathbb{Z}^{n}\right)\right\}} .
$$

Proposition 3.4 below states a similar result for affine systems. The result is a generalization of [14, Proposition 2.8], where the Bessel condition on $\mathcal{A}(\Psi)$ is relaxed by (2.19). Proposition 3.4 is a direct consequence of Propositions 2.5 and 2.7.

Proposition 3.4. Let $A \in G L_{n}(\mathbb{R})$ be expansive and $\Psi=\left\{\psi_{1}, \ldots, \psi_{L}\right\} \subset L^{2}\left(\mathbb{R}^{n}\right)$. Suppose that each $\psi \in \Psi$ satisfies condition (2.19). Then, for each $f \in \mathscr{D}$, the function

$$
w(x)=\sum_{g \in \mathcal{A}(\Psi)}\left|\left\langle T_{x} f, g\right\rangle\right|^{2}=\sum_{l=1}^{L} \sum_{j \in \mathbb{Z}} \sum_{k \in \mathbb{Z}^{n}}\left|\left\langle T_{x} f, D_{A^{j}} T_{k} \psi_{l}\right\rangle\right|^{2}
$$

is an almost periodic function that coincides pointwise with the absolutely convergent series

$$
w(x)=\sum_{l=1}^{L} \sum_{j \in \mathbb{Z}} \sum_{m \in \mathbb{Z}^{n}} c_{j, l}(m) \mathrm{e}^{2 \pi i\left\langle B^{j} m, x\right\rangle}
$$

where

$$
c_{j, l}(m)=\int_{\mathbb{R}^{n}} \hat{f}(\xi) \overline{\hat{f}\left(\xi+B^{j} m\right)} \overline{\hat{\psi}_{l}\left(B^{-j} \xi\right)} \hat{\psi}_{l}\left(B^{-j}\left(\xi+B^{j} m\right)\right) \mathrm{d} \xi .
$$


Remark 3. As noted in [14] the sum over $j \in \mathbb{Z}$ in Proposition 3.4 can be replaced by a sum over a smaller set $j \in \mathcal{J} \subset \mathbb{Z}$. The same holds for Proposition 3.3

The series representing $w$ and $w_{\Lambda}^{q}$ are very similar. By a change of variables, (3.14) becomes

$$
w_{\Lambda}^{q}(x)=\sum_{l=1}^{L} \sum_{j \in \mathbb{Z}} \sum_{m \in \mathbb{Z}^{n} \cap B^{-j} \Lambda^{*}} c_{j, l}(m) \mathrm{e}^{2 \pi i\left\langle B^{j} m, x\right\rangle},
$$

where the coefficients $c_{j, l}(m)$ are given by (3.19). Since $\mathbb{Z}^{n} \cap B^{-j} \Lambda^{*} \subset \mathbb{Z}^{n}$ for all $j \in \mathbb{Z}$, we can consider the series for $w_{\Lambda}^{q}$ in (3.20) as the series representing $w$ in (3.18) with some coefficients set to zero - exactly those coefficients $c_{j, l}(m)$ for which $m \in \mathbb{Z}^{n} \backslash B^{-j} \Lambda^{*}$. We stress that this connection holds without any assumptions on the rational lattice $\Lambda$, e.g., there is no assumption on $\Lambda$ being integer valued.

3.3. From affine to quasi-affine systems. The frame property carries over when moving from affine to $\Lambda$-oversampled quasi-affine systems for any rational lattice $\Lambda$. This statement is the main result of this section and is contained in Theorem 3.5 .

Theorem 3.5. Let $A \in G L_{n}(\mathbb{Q})$ be expansive, $\Psi \subset L^{2}\left(\mathbb{R}^{n}\right)$, and let $\Lambda$ be any rational lattice in $\mathbb{R}^{n}$. If the affine system $\mathcal{A}(\Psi)$ is a frame for $L^{2}\left(\mathbb{R}^{n}\right)$ with frame bounds $C_{1}, C_{2}$, then the $\Lambda$-oversampled quasi-affine system $\mathcal{A}_{\Lambda}^{q}(\Psi)$ is a frame for $L^{2}\left(\mathbb{R}^{n}\right)$ with frame bounds $C_{1}, C_{2}$.

The following lemma, which is needed in the proof of Theorem 3.5, is a consequence of [15, Lemma 23.19].

Lemma 3.6. Suppose $\mathrm{K}, \mathrm{M}$ are lattices in $\mathbb{R}^{n}$ such that $\mathrm{K} \subset \mathrm{M}$. Then, for $m \in \mathrm{K}^{*}$,

$$
\frac{1}{\#\{\mathrm{M} / \mathrm{K}\}} \sum_{d \in[\mathrm{M} / \mathrm{K}]} e^{2 \pi i\langle m, d\rangle}= \begin{cases}1, & m \in \mathrm{M}^{*}, \\ 0, & m \in \mathrm{K}^{*} \backslash \mathrm{M}^{*} .\end{cases}
$$

The proof of Theorem 3.5 relies on the following key result on translational averaging of affine functionals.

Lemma 3.7. Let $A \in G L_{n}(\mathbb{Q})$ be expansive, $\Psi \subset L^{2}\left(\mathbb{R}^{n}\right)$, and let $\Lambda$ be an integral lattice in $\mathbb{R}^{n}$. For each $J \in \mathbb{N}$ define

$$
\mathrm{M}_{J}=\bigcap_{|j| \leq J} A^{j} \mathbb{Z}^{n}
$$

Suppose the affine system $\mathcal{A}(\Psi)$ is a frame for $L^{2}\left(\mathbb{R}^{n}\right)$. Then

$$
N_{\Lambda}^{q}(f)=\lim _{J \rightarrow \infty} \frac{1}{\#\left\{\left(\mathrm{M}_{J}+\Lambda\right) / \mathrm{M}_{J}\right\}} \sum_{d \in\left[\left(\mathrm{M}_{J}+\Lambda\right) / \mathrm{M}_{J}\right]} N\left(T_{d} f\right) \quad \text { for } f \in \mathscr{D},
$$

where $\mathscr{D}$ is given by (1.6), $N$ by (3.5) and $N_{\Lambda}^{q}$ by (3.6).

Proof. Let $\Psi=\left\{\psi_{1}, \ldots, \psi_{L}\right\}$. For $f \in \mathscr{D}$, by (3.20),

$$
N_{\Lambda}^{q}(f)=w_{\Lambda}^{q}(0)=\sum_{l=1}^{L} \sum_{j \in \mathbb{Z}} \sum_{m \in \mathbb{Z}^{n} \cap B^{-j} \Lambda^{*}} c_{j, l}(m),
$$

where $c_{j, l}(m)$ are given in equation (3.19). So fix $J \in \mathbb{N}$ and let $\left\{d_{1}, \ldots, d_{s(J)}\right\}$ be a complete set of representatives of the quotient group $\left(\mathrm{M}_{J}+\Lambda\right) / \mathrm{M}_{J}$ so that $s(J)$ 
is the order of the group. We want to express $N_{\Lambda}^{q}(f)$ as an average of $N\left(T_{d_{r}} f\right)$ over $r=1, \ldots, s(J)$. Thus we consider

$$
\begin{aligned}
\frac{1}{s(J)} \sum_{r=1}^{s(J)} N\left(T_{d_{r}} f\right) & =\frac{1}{s(J)} \sum_{r=1}^{s(J)} \sum_{l=1}^{L} \sum_{|j| \leq J} \sum_{m \in \mathbb{Z}^{n}} c_{j, l}(m) \mathrm{e}^{2 \pi i\left\langle B^{j} m, d_{r}\right\rangle} \\
& +\frac{1}{s(J)} \sum_{r=1}^{s(J)} \sum_{l=1}^{L} \sum_{|j|>J} \sum_{m \in \mathbb{Z}^{n}} c_{j, l}(m) \mathrm{e}^{2 \pi i\left\langle B^{j} m, d_{r}\right\rangle} \\
& =: I_{1}(J)+I_{2}(J),
\end{aligned}
$$

which follows by (3.18). By absolute convergence of the sum above, we conclude that $I_{2}(J) \rightarrow 0$ as $J \rightarrow \infty$. Assume that the following identity holds:

$$
I_{1}(J)=\sum_{l=1}^{L} \sum_{|j| \leq J} \sum_{m \in \mathbb{Z}^{n} \cap B^{-j} \Lambda^{*}} c_{j, l}(m) .
$$

Taking the limit $J \rightarrow \infty$ in (3.24) and using equation (3.23) yield

$$
\begin{aligned}
\lim _{J \rightarrow \infty} \frac{1}{s(J)} \sum_{r=1}^{s(J)} N\left(T_{d_{r}} f\right)=\lim _{J \rightarrow \infty}\left(I_{1}(J)+I_{2}(J)\right) & =\lim _{J \rightarrow \infty} \sum_{l=1}^{L} \sum_{|j| \leq J} \sum_{m \in \mathbb{Z}^{n} \cap B^{-j} \Lambda^{*}} c_{j, l}(m) \\
& =N_{\Lambda}^{q}(f) .
\end{aligned}
$$

Hence, to complete the proof we only have left to show (3.25). Taking $\mathrm{K}=\mathrm{M}_{J}$ and $\mathrm{M}=\mathrm{M}_{J}+\Lambda$ in Lemma 3.6 gives us for all $\tilde{m} \in \mathrm{M}_{J}^{*}$ :

$$
\sum_{r=1}^{s(J)} e^{2 \pi i\left\langle\tilde{m}, d_{r}\right\rangle}= \begin{cases}s(J), & \tilde{m} \in \mathrm{M}_{J}^{*} \cap \Lambda^{*}, \\ 0, & \tilde{m} \in \mathrm{M}_{J}^{*} \backslash \Lambda^{*} .\end{cases}
$$

Fix $j \in \mathbb{Z}$ with $|j| \leq J$. Take $\tilde{m}=B^{j} m$. Obviously, $\tilde{m} \in \mathrm{M}_{J}^{*} \cap \Lambda^{*}$ precisely when $m \in B^{-j} \mathrm{M}_{J}^{*} \cap B^{-j} \Lambda^{*}$, and $\tilde{m} \in \mathrm{M}_{J}^{*} \backslash \Lambda^{*}$ precisely when $m \in B^{-j} \mathrm{M}_{J}^{*} \backslash B^{-j} \Lambda^{*}$. Since

$$
B^{-j} \mathrm{M}_{J}^{*}=\underset{-J-j \leq l \leq J-j}{+} B^{l} \mathbb{Z}^{n} \supset \mathbb{Z}^{n}
$$

we conclude from equation (3.26) that, for all $m \in \mathbb{Z}^{n}$,

$$
\sum_{r=1}^{s(J)} e^{2 \pi i\left\langle B^{j} m, d_{r}\right\rangle}= \begin{cases}s(J), & m \in \mathbb{Z}^{n} \cap B^{-j} \Lambda^{*}, \\ 0, & m \in \mathbb{Z}^{n} \backslash B^{-j} \Lambda^{*},\end{cases}
$$

and this holds for all $|j| \leq J$. Using these relations we arrive at

$$
\begin{aligned}
I_{1}(J) & \equiv \sum_{l=1}^{L} \sum_{|j| \leq J} \sum_{m \in \mathbb{Z}^{n}} c_{j, l}(m) \frac{1}{s(J)} \sum_{r=1}^{s(J)} \mathrm{e}^{2 \pi i\left\langle B^{j} m, d_{r}\right\rangle} \\
& =\sum_{l=1}^{L} \sum_{|j| \leq J} \sum_{m \in \mathbb{Z}^{n} \cap B^{-j} \Lambda^{*}} c_{j, l}(m),
\end{aligned}
$$

which completes the proof of the lemma.

Proof of Theorem 3.5. Assume that the affine system $\mathcal{A}(\Psi)$ is a frame for $L^{2}\left(\mathbb{R}^{n}\right)$ with bounds $C_{1}, C_{2}$. It suffices to prove that $\mathcal{A}_{\Lambda_{0}}^{q}(\Psi)$ is a frame for integer lattices $\Lambda_{0}$, i.e., $\Lambda_{0} \subset \mathbb{Z}^{n}$, which follows from the fact that any rational lattice $\Lambda$ has an 
integral sublattice of the form $c \mathbb{Z}^{n}$ for some $c \in \mathbb{N}$, e.g., take $c=d\left(\Lambda \cap \mathbb{Z}^{n}\right)$; see equation (2.5). Hence, if we prove that $\mathcal{A}_{c \mathbb{Z}^{n}}^{q}(\Psi)$ is a frame with bounds $C_{1}, C_{2}$, then, by applying Lemma [3.1, $\mathcal{A}_{\Lambda}^{q}(\Psi)$ is a frame with the frame bounds being preserved.

So let $\Lambda_{0}$ be an integral lattice. By our hypothesis there are constants $C_{1}, C_{2}>0$ so that

$$
C_{1}\|f\|^{2} \leq N(f) \leq C_{2}\|f\|^{2} \quad \forall f \in L^{2}\left(\mathbb{R}^{n}\right) .
$$

Fix $J \in \mathbb{N}$ and consider $\mathbf{M}_{J}$ introduced above. For each representative $d \in\left[\mathrm{M}_{J}+\right.$ $\left.\left.\Lambda_{0}\right) / \mathrm{M}_{J}\right]$ we have

$$
C_{1}\|f\|^{2} \leq N\left(T_{d} f\right) \leq C_{2}\|f\|^{2} \quad \forall f \in L^{2}\left(\mathbb{R}^{n}\right),
$$

where we have used the fact that $\left\|T_{x} f\right\|=\|f\|$ for $x \in \mathbb{R}^{n}$. Adding these equations for each representative $d$ yields

$$
\#\left\{\left(\mathrm{M}_{J}+\Lambda_{0}\right) / \mathrm{M}_{J}\right\} C_{1}\|f\|^{2} \leq \sum_{d \in\left[\left(\mathrm{M}_{J}+\Lambda_{0}\right) / \mathrm{M}_{J}\right]} N\left(T_{d} f\right) \leq \#\left\{\left(\mathrm{M}_{J}+\Lambda_{0}\right) / \mathrm{M}_{J}\right\} C_{2}\|f\|^{2} .
$$

By taking the limit $J \rightarrow \infty$, we have

$$
C_{1}\|f\|^{2} \leq \lim _{J \rightarrow \infty} \frac{1}{\#\left\{\left(\mathrm{M}_{J}+\Lambda_{0}\right) / \mathrm{M}_{J}\right\}} \sum_{d \in\left[\left(\mathrm{M}_{J}+\Lambda_{0}\right) / \mathrm{M}_{J}\right]} N\left(T_{d} f\right) \leq C_{2}\|f\|^{2}
$$

for all $f \in L^{2}\left(\mathbb{R}^{n}\right)$. Since $\Lambda_{0}$ is an integer lattice, we can apply Lemma 3.7. This gives us

$$
C_{1}\|f\|^{2} \leq N_{\Lambda}^{q}(f) \leq C_{2}\|f\|^{2}
$$

for $f \in \mathscr{D}$. Extending these inequalities to all of $L^{2}\left(\mathbb{R}^{n}\right)$ by a standard density argument completes the proof.

Remark 4. The special case of Theorem 3.5 in one dimension with $\Lambda=\mathbb{Z}$ was first shown in [14, Theorem 2.18]. In fact, [14, Theorem 2.18] is stated for quasi-affine systems obtained by oversampling with respect to the lattice $\Lambda=s^{-1} \mathbb{Z}$, where $s$ is relatively prime to $p$ and $q$, and $a=p / q$ is a dilation factor. In this case the quasi-affine system $\mathcal{A}_{\Lambda}^{q}(\Psi)$ takes a nice algebraic form:

$$
\mathcal{A}_{s^{-1} \mathbb{Z}}^{q}(\Psi)=\left\{\begin{array}{ll}
|p|^{j / 2}|q|^{-j}|s|^{-1 / 2} \psi\left(a^{j} x-s^{-1} q^{-j} k\right): & j \geq 0, k \in \mathbb{Z} \\
|p|^{j}|q|^{-j / 2}|s|^{-1 / 2} \psi\left(a^{j} x-s^{-1} p^{j} k\right): & j<0, k \in \mathbb{Z}
\end{array}\right\} .
$$

Hence, the above system is obtained by further oversampling of the standard quasiaffine system $\mathcal{A}_{\mathbb{Z}}^{q}(\Psi)$ given by (1.3). However, our Theorem 3.5 holds for oversampling with respect to any rational lattice $\Lambda$, such as in (1.4) or in Example 3 . The sparser the lattice $\Lambda$ is, the better result we have due to Lemma 3.1 on oversampling of quasi-affine systems.

3.4. From quasi-affine to affine systems. When moving from quasi-affine to affine systems the frame property only carries over if we impose stronger conditions on the set of generators. Hence, we have only the following partial converse of Theorem 3.5.

Theorem 3.8. Let $A \in G L_{n}(\mathbb{Q})$ be expansive and $\Psi \subset L^{2}\left(\mathbb{R}^{n}\right)$. If the $\mathrm{M}_{J^{-}}$ oversampled quasi-affine system $\mathcal{A}_{\mathrm{M}_{J}}^{q}(\Psi)$ is a frame for $L^{2}\left(\mathbb{R}^{n}\right)$ with uniform frame bounds $C_{1}, C_{2}$ for all $J \in \mathbb{N}$, where $\mathrm{M}_{J}$ is given by (3.4), then the affine system $\mathcal{A}(\Psi)$ is a frame for $L^{2}\left(\mathbb{R}^{n}\right)$ with frame bounds $C_{1}, C_{2}$. 
Proof. Assume that

$$
C_{1}\|f\|^{2} \leq N_{\mathrm{M}_{J}}^{q}(f) \leq C_{2}\|f\|^{2} \quad \text { for all } f \in \mathscr{D}
$$

holds for all $J \in \mathbb{N}$. Since scale $j$ of the affine system and the $\mathrm{M}_{J}$-oversampled quasi-affine system agree whenever $|j| \leq J$, we have by (3.7),

$$
K_{j}(f)=K_{\mathrm{M}_{J}, j}^{q}(f) \quad \text { for all }|j| \leq J, f \in L^{2}\left(\mathbb{R}^{n}\right) .
$$

Thus, for $J \in \mathbb{N}$,

$$
\sum_{|j| \leq J} K_{j}(f)=\sum_{|j| \leq J} K_{\mathrm{M}_{J}, j}^{q}(f) \leq C_{2}\|f\|^{2} .
$$

Letting $J \rightarrow \infty$ yields

$$
N(f)=\lim _{J \rightarrow \infty} \sum_{|j| \leq J} K_{j}(f) \leq \limsup _{J \rightarrow \infty} \sum_{|j| \leq J} K_{\mathrm{M}_{J}, j}^{q}(f) \leq C_{2}\|f\|^{2},
$$

whereby we conclude that $\mathcal{A}(\Psi)$ is a Bessel sequence with bound $C_{2}$. Likewise for the lower bound:

$$
C_{1}\|f\|^{2} \leq \sum_{|j| \leq J} K_{\mathrm{M}_{J}, j}^{q}(f)+\sum_{|j|>J} K_{\mathrm{M}_{J}, j}^{q}(f)=\sum_{|j| \leq J} K_{j}(f)+\sum_{|j|>J} K_{\mathrm{M}_{J}, j}^{q}(f) .
$$

Suppose that

$$
\lim _{J \rightarrow \infty} \sum_{|j|>J} K_{\mathrm{M}_{J}, j}^{q}(f)=0 \quad \text { for } f \in \mathscr{D} .
$$

Then, by equation (3.28),

$$
C_{1}\|f\|^{2} \leq \lim _{J \rightarrow \infty} \sum_{|j| \leq J} K_{j}(f)=N(f) \quad \text { for } f \in \mathscr{D} .
$$

Since $\mathcal{A}(\Psi)$ satisfies the upper bound, we can extend this inequality to all of $L^{2}\left(\mathbb{R}^{n}\right)$ by a density argument. Hence the affine system $\mathcal{A}(\Psi)$ satisfies the lower bound with constant $C_{1}$.

To complete the proof we need to verify (3.29). We have already showed that $\mathcal{A}(\Psi)$ is a Bessel sequence, so by Proposition 3.4 the series in (3.18) converges absolutely and

$$
\sum_{l=1}^{L} \sum_{j \in \mathbb{Z}} \sum_{m \in \mathbb{Z}^{n}}\left|c_{j, l}(m)\right|<\infty,
$$

where $c_{j, l}(m)$ is given by (3.19). Therefore, by (3.20) and Remark 3 ,

$$
\begin{aligned}
\sum_{|j|>J} K_{\mathrm{M}_{J}, j}^{q}(f) & \equiv \sum_{|j|>J} \sum_{l=1}^{L} \sum_{k \in \mathrm{K}_{j}}\left|\left\langle f, d_{j} T_{k} D_{A^{j}} \psi_{l}\right\rangle\right|^{2} \leq \sum_{|j|>J} \sum_{l=1}^{L} \sum_{m \in \mathbb{Z}^{n} \cap B^{-j} \mathrm{M}_{J}^{*}}\left|c_{j, l}(m)\right| \\
& \leq \sum_{|j|>J} \sum_{l=1}^{L} \sum_{m \in \mathbb{Z}^{n}}\left|c_{j, l}(m)\right| \rightarrow 0 \quad \text { as } J \rightarrow \infty .
\end{aligned}
$$

This shows (3.29) and completes the proof of Theorem 3.8 .

The following result combines Theorems 3.5 and 3.8 in a more conceptually transparent and less technical form. 
Theorem 3.9. Let $A \in G L_{n}(\mathbb{Q})$ be expansive and $\Psi \subset L^{2}\left(\mathbb{R}^{n}\right)$. Then, the affine system $\mathcal{A}(\Psi)$ is a frame for $L^{2}\left(\mathbb{R}^{n}\right)$ with frame bounds $C_{1}, C_{2}$ if, and only if, the $\Lambda$-oversampled quasi-affine system $\mathcal{A}_{\Lambda}^{q}(\Psi)$ is a frame for $L^{2}\left(\mathbb{R}^{n}\right)$ with uniform frame bounds $C_{1}, C_{2}$ for all integer lattices $\Lambda$.

3.5. Recovering known equivalence results. We end this section by illustrating the general nature of Theorems 3.5 and 3.8. In particular, we will show that the well-known equivalence result of Ron and Shen [20] for affine and quasi-affine frames for integer dilation $A \in G L_{n}(\mathbb{Z})$ is a simple consequence of these results. Moreover, we have the following generalization of their result.

Proposition 3.10. Let $A \in G L_{n}(\mathbb{Z})$ be expansive and $\Psi \subset L^{2}(\mathbb{R})$. Then the following assertions are equivalent:

(i) $\mathcal{A}(\Psi)$ is a frame with bounds $C_{1}, C_{2}$,

(ii) $\mathcal{A}_{\Lambda_{0}}^{q}(\Psi)$ is a frame with bounds $C_{1}, C_{2}$ for some oversampling lattice $\Lambda_{0} \subset \mathbb{Z}^{n}$,

(iii) $\mathcal{A}_{\Lambda}^{q}(\Psi)$ is a frame with bounds $C_{1}, C_{2}$ for all oversampling lattices $\Lambda \subset \mathbb{Z}^{n}$.

Proof. By Theorem 3.5. we are only left to prove (ii) $\Rightarrow$ (i), but this will follow from an application of Theorem 3.8 From Lemma 3.1 we have that $\mathcal{A}^{q}(\Psi)$ is a frame for $L^{2}\left(\mathbb{R}^{n}\right)$ with bounds $C_{1}, C_{2}$. Recall the identity

$$
\mathcal{A}_{A^{l} \mathbb{Z}^{n}}^{q}(\Psi)=D_{A^{-l}}\left(\mathcal{A}^{q}(\Psi)\right) \quad \text { for } l \in \mathbb{Z}
$$

from Example 2. This tells us, by unitarity of the dilation operator, that $\mathcal{A}_{A^{l} \mathbb{Z}^{n}}^{q}(\Psi)$ is a frame with (uniform) bounds $C_{1}, C_{2}$ for each $l \in \mathbb{Z}$. Since $A$ has integer entries, we have

$$
\mathrm{M}_{J} \equiv \bigcap_{|j| \leq J} A^{j} \mathbb{Z}^{n}=A^{J} \mathbb{Z}^{n} \quad \text { for } J \in \mathbb{N}
$$

and the conclusion follows from Theorem 3.8 ,

\section{DUAL AFFINE AND QUASI-AFFINE FRAMES}

The goal of this section is to prove the equivalence between pairs of dual affine and quasi-affine frames in the setting of rational dilations. To achieve this we will use well-studied fundamental equations of affine systems.

Definition 4.1. Suppose that $\Psi=\left\{\psi_{1}, \ldots, \psi_{L}\right\} \subset L^{2}\left(\mathbb{R}^{n}\right)$ and $\Phi=\left\{\phi_{1}, \ldots, \phi_{L}\right\}$ $\subset L^{2}\left(\mathbb{R}^{n}\right)$ are such that

$$
\sum_{l=1}^{L} \sum_{j \in \mathbb{Z}}\left(\left|\hat{\psi}_{l}\left(B^{-j} \xi\right)\right|^{2}+\left|\hat{\phi}_{l}\left(B^{-j} \xi\right)\right|^{2}\right)<\infty \quad \text { for a.e. } \xi .
$$

We say that a pair $(\Psi, \Phi)$ satisfies the fundamental equations if

$$
\tilde{t}_{\alpha}(\xi):=\sum_{l=1}^{L} \sum_{j \in \mathbb{Z}: \alpha \in B^{j} \mathbb{Z}^{n}} \hat{\psi}_{l}\left(B^{-j} \xi\right) \overline{\hat{\phi}_{l}\left(B^{-j}(\xi+\alpha)\right)}=0 \quad \text { for a.e. } \xi
$$

$$
\text { and all } \alpha \in \mathbb{Z}^{n} \backslash\{0\} \text {. }
$$


Remark 5. Note that the assumption (4.1) is made to guarantee that the series in (4.2) converges absolutely, and hence the Calderón condition (4.2) is meaningful. On the other hand, the series (4.3) converges absolutely for a.e. $\xi$ without any assumptions (apart from $\Psi, \Phi \subset L^{2}\left(\mathbb{R}^{n}\right)$, that is). Indeed, for any $\psi \in L^{2}\left(\mathbb{R}^{n}\right)$ and $\alpha \in \mathbb{R}^{n}$,

$$
\int_{\mathbb{R}^{n}} \sum_{j \leq J}\left|\hat{\psi}\left(B^{-j}(\xi+\alpha)\right)\right|^{2} \mathrm{~d} \xi=\int_{\mathbb{R}^{n}} \sum_{j \leq J}|\operatorname{det} A|^{j}|\hat{\psi}(\xi)|^{2} \mathrm{~d} \xi=\frac{|\operatorname{det} A|^{J+1}}{|\operatorname{det} A|-1}\|\psi\|^{2}<\infty
$$

for any $J \in \mathbb{N}$. Since the dilation $B$ is expansive, for any $\alpha \neq 0$, there exists $J \in \mathbb{N}$ such that $j \in \mathbb{Z}$ and $\alpha \in B^{j} \mathbb{Z}^{n}$ implies that $j \leq J$. Hence, by $2|z w| \leq|z|^{2}+|w|^{2}$ for $z, w \in \mathbb{C}$,

$$
\begin{aligned}
\sum_{j \in \mathbb{Z}: \alpha \in B^{j} \mathbb{Z}^{n}}\left|\hat{\psi}_{l}\left(B^{-j} \xi\right) \overline{\hat{\phi}_{l}\left(B^{-j}(\xi+\alpha)\right)}\right| \leq \frac{1}{2} \sum_{j \in \mathbb{Z}: \alpha \in B^{j} \mathbb{Z}^{n}}\left|\hat{\psi}_{l}\left(B^{-j} \xi\right)\right|^{2} \\
+\frac{1}{2} \sum_{j \in \mathbb{Z}: \alpha \in B^{j} \mathbb{Z}^{n}}\left|\hat{\phi}_{l}\left(B^{-j}(\xi+\alpha)\right)\right|^{2}<\infty \quad \text { for a.e. } \xi \in \mathbb{R}^{n} .
\end{aligned}
$$

The last inequality is a consequence of (4.4).

We will need the following result which was originally proved by Frazier, Garrigós, Wang, and Weiss [11] in the dyadic setting. Later it was extended by the first author [2] to the setting of integer, expansive dilations and by Chui, Czaja, Maggioni, and Weiss 8 to the setting of real, expansive dilations. We include an alternative proof of Theorem 4.1 for the sake of completeness and since its techniques will be used later.

Theorem 4.1. Let $A \in G L_{n}(\mathbb{R})$ be expansive. Suppose that $\Psi=\left\{\psi_{1}, \ldots, \psi_{L}\right\} \subset$ $L^{2}\left(\mathbb{R}^{n}\right)$ and $\Phi=\left\{\phi_{1}, \ldots, \phi_{L}\right\} \subset L^{2}\left(\mathbb{R}^{n}\right)$ are such that

$$
\sum_{l=1}^{L} \sum_{j \in \mathbb{Z}}\left(\left|\hat{\psi}_{l}\left(B^{-j} \xi\right)\right|^{2}+\left|\hat{\phi}_{l}\left(B^{-j} \xi\right)\right|^{2}\right) \in L_{\mathrm{loc}}^{1}\left(\mathbb{R}^{n} \backslash\{0\}\right) .
$$

Then, the affine systems $\mathcal{A}(\Psi)$ and $\mathcal{A}(\Phi)$ form a weak pair of frames, i.e.,

$$
\|f\|^{2}=\sum_{l=1}^{L} \sum_{j \in \mathbb{Z}} \sum_{k \in \mathbb{Z}^{n}}\left\langle f, D_{A^{j}} T_{k} \psi_{l}\right\rangle\left\langle D_{A^{j}} T_{k} \phi_{l}, f\right\rangle \quad \text { for all } f \in \mathscr{D},
$$

if, and only if, the fundamental equations (4.2) and (4.3) hold.

Proof. The proof is based on Proposition 3.4 on affine systems and the idea of polarization as in [18, Section 8]. By our assumption on $\Psi$ and $\Phi$, we can define

$$
N(f, \Psi, \Phi)=\sum_{l=1}^{L} \sum_{j \in \mathbb{Z}} \sum_{k \in \mathbb{Z}^{n}}\left\langle f, D_{A^{j}} T_{k} \psi_{l}\right\rangle\left\langle D_{A^{j}} T_{k} \phi_{l}, f\right\rangle \quad \text { for } f \in \mathscr{D},
$$

where the multiple series converge absolutely. This follows immediately by Remark 1 and

$$
2\left|\left\langle f, D_{A^{j}} T_{k} \psi_{l}\right\rangle\left\langle D_{A^{j}} T_{k} \phi_{l}, f\right\rangle\right| \leq\left|\left\langle f, D_{A^{j}} T_{k} \psi_{l}\right\rangle\right|^{2}+\left|\left\langle D_{A^{j}} T_{k} \phi_{l}, f\right\rangle\right|^{2} .
$$


By the polarization identity

$$
\bar{z} w=\frac{1}{4} \sum_{p=1}^{4} i^{p}\left|i^{p} z+w\right|^{2} \quad \text { for } z, w \in \mathbb{C},
$$

we have

$$
N(f, \Psi, \Phi)=\frac{1}{4} \sum_{p=1}^{4} i^{p} N\left(f, \Theta_{p}\right), \quad \text { where } \Theta_{p}=\left\{\theta_{l, p}\right\}_{l=1}^{L}, \theta_{l, p}=i^{p} \psi_{l}+\phi_{l}
$$

for $f \in \mathscr{D}$.

Since, for $p=1,2,3,4$,

$$
\begin{aligned}
\sum_{l=1}^{L} \sum_{j \in \mathbb{Z}}\left|\hat{\theta}_{l, p}\left(B^{-j} \xi\right)\right|^{2} & \equiv \sum_{l=1}^{L} \sum_{j \in \mathbb{Z}}\left|i^{p} \hat{\psi}_{l}\left(B^{-j} \xi\right)+\hat{\phi}_{l}\left(B^{-j} \xi\right)\right|^{2} \\
& \leq 2 \sum_{l=1}^{L} \sum_{j \in \mathbb{Z}}\left(\left|\hat{\psi}_{l}\left(B^{-j} \xi\right)\right|^{2}+\left|\hat{\phi}_{l}\left(B^{-j} \xi\right)\right|^{2}\right) \in L_{\mathrm{loc}}^{1}\left(\mathbb{R}^{n} \backslash\{0\}\right),
\end{aligned}
$$

we can apply Proposition 3.4 to $\Theta_{p}$ for each $p$. This yields

$$
N\left(T_{x} f, \Theta_{p}\right)=\sum_{l=1}^{L} \sum_{j \in \mathbb{Z}} \sum_{m \in \mathbb{Z}^{n}} b_{j, l, p}(m) \mathrm{e}^{2 \pi i\left\langle B^{j} m, x\right\rangle},
$$

where

$$
b_{j, l, p}(m)=\int_{\mathbb{R}^{n}} \hat{f}(\xi) \overline{\hat{f}\left(\xi+B^{j} m\right)} \overline{\hat{\theta}_{l, p}\left(B^{-j} \xi\right)} \hat{\theta}_{l, p}\left(B^{-j}\left(\xi+B^{j} m\right)\right) \mathrm{d} \xi
$$

for $l=1, \ldots, L, j \in \mathbb{Z}, m \in \mathbb{Z}^{n}$, and the integral in (4.8) converges absolutely. By the polarization identity

$$
\overline{z_{1}} w_{2}=\frac{1}{4} \sum_{p=1}^{4} i^{p} \overline{\left(i^{p} z_{1}+w_{1}\right)}\left(i^{p} z_{2}+w_{2}\right) \quad \text { for } z_{1}, z_{2}, w_{1}, w_{2} \in \mathbb{C},
$$

we have

$$
\begin{aligned}
& \frac{1}{4} \sum_{p=1}^{4} i^{p} \overline{\hat{\theta}_{l, p}\left(B^{-j} \xi\right)} \hat{\theta}_{l, p}\left(B^{-j}\left(\xi+B^{j} m\right)\right) \\
& \equiv \frac{1}{4} \sum_{p=1}^{4} i^{p} \overline{\left(i^{p} \hat{\psi}_{l}\left(B^{-j} \xi\right)+\hat{\phi}_{l}\left(B^{-j} \xi\right)\right)}\left(i^{p} \hat{\psi}_{l}\left(B^{-j}\left(\xi+B^{j} m\right)\right)+\hat{\phi}_{l}\left(B^{-j}\left(\xi+B^{j} m\right)\right)\right) \\
& =\overline{\hat{\psi}_{l}\left(B^{-j} \xi\right)} \hat{\phi}_{l}\left(B^{-j}\left(\xi+B^{j} m\right)\right) .
\end{aligned}
$$

Therefore, by (4.7),

$$
\tilde{w}(x):=N\left(T_{x} f, \Psi, \Phi\right)=\sum_{l=1}^{L} \sum_{j \in \mathbb{Z}} \sum_{m \in \mathbb{Z}^{n}} \tilde{c}_{j, l}(m) \mathrm{e}^{2 \pi i\left\langle B^{j} m, x\right\rangle},
$$

where

$$
\tilde{c}_{j, l}(m)=\frac{1}{4} \sum_{p=1}^{4} i^{p} b_{j, l, p}(m)=\int_{\mathbb{R}^{n}} \hat{f}(\xi) \overline{\hat{f}\left(\xi+B^{j} m\right)} \overline{\hat{\psi}_{l}\left(B^{-j} \xi\right)} \hat{\phi}_{l}\left(B^{-j}\left(\xi+B^{j} m\right)\right) \mathrm{d} \xi .
$$


By a change of summation order, using absolute convergence of the series in (4.9), we have

$$
\tilde{w}(x)=\sum_{\alpha \in \bigcup_{j \in \mathbb{Z}} B^{j} \mathbb{Z}^{n}} \tilde{c}_{\alpha} \mathrm{e}^{2 \pi i\langle\alpha, x\rangle},
$$

where

$$
\begin{aligned}
& \tilde{c}_{\alpha}=\int_{\mathbb{R}^{n}} \hat{f}(\xi) \overline{\hat{f}(\xi+\alpha)} \sum_{l=1}^{L} \sum_{j \in \mathbb{Z}: \alpha \in B^{j} \mathbb{Z}^{n}} \overline{\hat{\psi}_{l}\left(B^{-j} \xi\right)} \hat{\phi}_{l}\left(B^{-j}(\xi+\alpha)\right) \mathrm{d} \xi \\
& =\int_{\mathbb{R}^{n}} \hat{f}(\xi) \overline{\hat{f}(\xi+\alpha) \tilde{t}_{\alpha}(\xi)} \mathrm{d} \xi, \quad \text { for } \alpha \in \bigcup_{j \in \mathbb{Z}} B^{j} \mathbb{Z}^{n} .
\end{aligned}
$$

Assume that the affine systems $\mathcal{A}(\Psi)$ and $\mathcal{A}(\Phi)$ form a weak pair of frames. Using $\left\|T_{x} f\right\|=\|f\|$, this implies that the almost periodic function $\tilde{w}(x)$ from (4.9) is constant. To be precise, $\tilde{w}(x)=\|f\|^{2}$. By uniqueness of coefficients for Fourier series of almost periodic functions [13. Lemma 2.5], this only happens if, for $\alpha \in \bigcup_{j \in \mathbb{Z}} B^{j} \mathbb{Z}^{n}$,

$$
\tilde{c}_{0}=\|f\|^{2} \quad \text { and } \quad \tilde{c}_{\alpha}=0 \quad \text { for } \alpha \neq 0 .
$$

By (4.11), this shows that

$$
\int_{\mathbb{R}^{n}}|\hat{f}(\xi)|^{2} \tilde{t}_{0}(\xi) \mathrm{d} \xi=\|f\|^{2}=\|\hat{f}\|^{2} \quad \text { for all } f \in \mathscr{D} .
$$

Since $\mathscr{D}$ is dense in $L^{2}\left(\mathbb{R}^{n}\right)$, this implies further that $\tilde{t}_{0}(\xi)=1$ for a.e. $\xi \in \mathbb{R}^{n}$ showing that the first fundamental equation (4.2) holds.

For a non-zero $\alpha$ we have by (4.11) and (4.12),

$$
\int_{\mathbb{R}^{n}} \hat{f}(\xi) \overline{\hat{f}(\xi+\alpha) \tilde{t}_{\alpha}(\xi)} \mathrm{d} \xi=0 \quad \text { for all } f \in \mathscr{D}
$$

for $\alpha \in\left(\bigcup_{j \in \mathbb{Z}} B^{j} \mathbb{Z}^{n}\right) \backslash\{0\}$. In particular, this equality holds for $\alpha \in \mathbb{Z}^{n} \backslash\{0\}$. We need to show that $\tilde{t}_{\alpha}=0$ almost everywhere for $\alpha \in \mathbb{Z}^{n} \backslash\{0\}$. The conclusion is almost immediate from Du Bois-Reynold's lemma, which says that for local integrable functions $u$ on $\mathbb{R}^{n}$ satisfying $\int u v=0$ for all $v \in C_{0}^{\infty}$ we have $u=0$. We fix $\alpha \in \mathbb{Z}^{n} \backslash\{0\}$, and let $I_{\mathbb{Z}^{n}}$ denote a fundamental domain of $\mathbb{Z}^{n}$. For arbitrary $l \in \mathbb{Z}^{n}$ we consider the translated parallelotope $I_{l}=I_{\mathbb{Z}^{n}}+l \subset \mathbb{R}^{n}$ and define $f$ by

$$
\hat{f}(\xi)= \begin{cases}1 & \text { for } \xi \in I_{l}, \\ \overline{\tilde{t}_{\alpha}(\xi)} & \text { for } \xi+\alpha \in I_{l}, \\ 0 & \text { otherwise. }\end{cases}
$$

This definition makes sense since $\bigcup_{l \in \mathbb{Z}^{n}} I_{l}=\mathbb{R}^{n}$ and $\left(I_{l}-\alpha\right) \cap I_{l}=\emptyset$ for $\alpha \in \mathbb{Z}^{n} \backslash\{0\}$. Furthermore, since $\tilde{t}_{\alpha}$ is bounded by Remark 5 , we have $f \in \mathscr{D}$. Consequently,

$$
0=\int_{\mathbb{R}^{n}} \hat{f}(\xi) \overline{\hat{f}(\xi+\alpha) \tilde{t}_{\alpha}(\xi)} \mathrm{d} \xi=\int_{I_{l}} 1 \tilde{t}_{\alpha}(\xi) \overline{\tilde{t}_{\alpha}(\xi)} \mathrm{d} \xi=\int_{I_{l}}\left|\tilde{t}_{\alpha}(\xi)\right|^{2} \mathrm{~d} \xi
$$

which implies that $\tilde{t}_{\alpha}(\xi)$ vanishes almost everywhere for $\xi \in I_{l}$. Since $l \in \mathbb{Z}^{n}$ was arbitrarily chosen, we deduce that $\tilde{t}_{\alpha}(\xi)=0$ for a.e. $\xi \in \mathbb{R}^{n}$. This shows that the second fundamental equation (4.3) holds.

Conversely, assume that the fundamental equations (4.2) and (4.3) hold. Equation (4.3) states that $\tilde{t}_{\alpha}(\xi)=0$ for a.e. $\xi \in \mathbb{R}^{n}$ for $\alpha \in \mathbb{Z}^{n} \backslash\{0\}$. By a change of 
variables $\gamma=B^{l} \xi$ and $\beta=B^{l} \alpha(l \in \mathbb{Z})$, this implies $\tilde{t}_{\beta}(\gamma)=0$ for $\beta \in B^{l} \mathbb{Z}^{n} \backslash\{0\}$. Since this holds for all $l \in \mathbb{Z}$, we conclude $\tilde{t}_{\alpha}=0$ almost everywhere for $\alpha \in$ $\bigcup_{j \in \mathbb{Z}} B^{j} \mathbb{Z}^{n} \backslash\{0\}$. Hence, by (4.11), $\tilde{c}_{\alpha}=0$ for $\alpha \in \bigcup_{j \in \mathbb{Z}} B^{j} \mathbb{Z}^{n} \backslash\{0\}$. Therefore, $\tilde{w}(x)=\tilde{c}_{0}=\|f\|^{2}$ for all $x \in \mathbb{R}^{n}$, so, in particular,

$$
N(f, \Psi, \Phi) \equiv \tilde{w}(0)=\|f\|^{2} \quad \text { for all } f \in \mathscr{D} .
$$

We conclude that the affine systems $\mathcal{A}(\Psi)$ and $\mathcal{A}(\Phi)$ form a weak pair of frames.

We are now able to prove the characterization of dual affine and quasi-affine frames in terms of fundamental equations using the theory of mixed dual Gramians of Ron and Shen [19, 21, 23. An alternative proof using the ideas of polarization of affine functionals is presented at the end of this section. In the integer case Theorem 4.2 was first shown by Ron and Shen 20,22 with some decay assumptions on generators $\Psi$ and $\Phi$. Chui, Shi, and Stöckler 9 proved the same result without any decay assumptions; see also [2, Theorem 4.1]. Theorem 4.2 generalizes this result to the setting of rational dilations.

Theorem 4.2. Let $A \in G L_{n}(\mathbb{Q})$ be expansive. Suppose $\mathcal{A}(\Psi)$ and $\mathcal{A}(\Phi)$ are Bessel sequences in $L^{2}\left(\mathbb{R}^{n}\right)$. Then the following assertions are equivalent:

(i) $\mathcal{A}(\Psi)$ and $\mathcal{A}(\Phi)$ are dual frames.

(ii) $\mathcal{A}_{\Lambda_{0}}^{q}(\Psi)$ and $\mathcal{A}_{\Lambda_{0}}^{q}(\Phi)$ are dual frames for some integer oversampling lattice $\Lambda_{0} \subset \mathbb{Z}^{n}$.

(iii) $\mathcal{A}_{\Lambda}^{q}(\Psi)$ and $\mathcal{A}_{\Lambda}^{q}(\Phi)$ are dual frames for all integer oversampling lattices $\Lambda \subset$ $\mathbb{Z}^{n}$.

(iv) $\Psi$ and $\Phi$ satisfy the fundamental equations (4.2) and (4.3).

Proof. The local integrability condition in Theorem 4.1 is satisfied by Proposition 3.2 since $\mathcal{A}(\Psi)$ and $\mathcal{A}(\Phi)$ are assumed to be Bessel sequences. Furthermore, weak duality (4.5) of two Bessel sequences implies "strong" duality [2, Lemma 2.7], i.e., that $\mathcal{A}(\Psi)$ and $\mathcal{A}(\Phi)$ are dual frames. Hence, by Theorem 4.1, we have (i) $\Leftrightarrow$ (iv); this equivalence is well known, even for real dilations [ 8 , Theorem 4].

The proof of the equivalences (ii) $\Leftrightarrow$ (iii) $\Leftrightarrow$ (iv) is based on the approach used in 3. Theorem 3.4]. Let $\widetilde{G}_{j}(\xi)_{k, l}$ denote the mixed dual Gramian of $O_{\Lambda}^{A^{-j} \mathbb{Z}^{n}}\left(D_{A^{j}} \Psi\right)$ and $O_{\Lambda}^{A^{-j} \mathbb{Z}^{n}}\left(D_{A^{j}} \Phi\right)$ for $j \in \mathbb{Z}$; see Section 2.4. By Lemma 2.3 with $\Gamma=A^{-j} \mathbb{Z}^{n}$, this mixed dual Gramian is given as

$$
\begin{aligned}
\widetilde{G}_{j}(\xi)_{k, l} & = \begin{cases}|\operatorname{det} A|^{j} \sum_{l=1}^{L} \widehat{D_{A^{j}} \psi}(\xi+k) \overline{\widehat{D_{A^{j}} \phi}(\xi+l)}, & k-l \in \Gamma^{*} \cap \Lambda^{*}, \\
0, & k-l \in \Lambda^{*} \backslash \Gamma^{*},\end{cases} \\
& = \begin{cases}\sum_{l=1}^{L} \hat{\psi}_{l}(\xi+k) \overline{\hat{\phi}_{l}(\xi+l)}, & k-l \in B^{j} \mathbb{Z}^{n} \cap \Lambda^{*}, \\
0, & k-l \in \Lambda^{*} \backslash B^{j} \mathbb{Z}^{n},\end{cases}
\end{aligned}
$$

for $k, l \in \Lambda^{*}$. The mixed dual Gramian of $\mathcal{A}_{\Lambda}^{q}(\Psi)$ and $\mathcal{A}_{\Lambda}^{q}(\Phi)$ is found by additivity of the $j$ th layer mixed dual Gramian $\widetilde{G}_{j}(\xi)$ as

$$
\begin{aligned}
\widetilde{G}(\xi)_{k, l} & =\sum_{j \in \mathbb{Z}} \widetilde{G}_{j}(\xi)_{k, l} \\
& =\sum_{l=1}^{L} \sum_{j \in \mathbb{Z}} \hat{\psi}_{l}\left(B^{-j}(\xi+k)\right) \overline{\hat{\phi}_{l}\left(B^{-} j(\xi+l)\right)} \times \begin{cases}1, & k-l \in B^{j} \mathbb{Z}^{n} \cap \Lambda^{*}, \\
0, & k-l \in \Lambda^{*} \backslash B^{j} \mathbb{Z}^{n},\end{cases}
\end{aligned}
$$


for $k, l \in \Lambda^{*}$. We only consider $k, l \in \Lambda^{*}$, so $k-l \in \Lambda^{*}$ is trivially satisfied. Thus, we arrive at the following expression for the mixed dual Gramian:

$$
\widetilde{G}(\xi)_{k, l}=\sum_{l=1}^{L} \sum_{j \in \mathbb{Z}: k-l \in B^{j} \mathbb{Z}^{n}} \hat{\psi}_{l}\left(B^{-j}(\xi+k)\right) \overline{\hat{\phi}_{l}\left(B^{-} j(\xi+l)\right)} \equiv \tilde{t}_{l-k}(\xi+k) .
$$

Assume (ii) holds. This implies that the mixed dual Gramian $\widetilde{G}(\xi)$ is the identity operator on $\ell^{2}\left(\Lambda_{0}^{*}\right)$ for a.e. $\xi \in I_{\Lambda_{0}^{*}}$; hence $\widetilde{G}(\xi)_{k, l}=\delta_{k, l}$ for a.e. $\xi \in I_{\Lambda_{0}^{*}}$. By equation (4.13), for $\alpha \in \Lambda_{0}^{*}$,

$$
\delta_{\alpha, 0}=\sum_{l=1}^{L} \sum_{j \in \mathbb{Z}: \alpha \in B^{j} \mathbb{Z}^{n}} \hat{\psi}_{l}\left(B^{-j} \xi\right) \overline{\hat{\phi}_{l}\left(B^{-j}(\xi+\alpha)\right)} \equiv \tilde{t}_{\alpha}(\xi) \quad \text { for a.e. } \xi \in \mathbb{R}^{n} .
$$

This implies (iv) since $\mathbb{Z}^{n} \subset \Lambda_{0}^{*}$.

Assume (iv) holds. We will show that this implies (iii), i.e., that $\widetilde{G}(\xi)_{k, l}=\delta_{k, l}$ for a.e. $\xi \in I_{\Lambda^{*}}$ and all $k, l \in \Lambda^{*}$, where $\Lambda$ is any integer lattice satisfying $\Lambda \subset \mathbb{Z}^{n}$. By a change of variables, we see that $\tilde{t}_{\alpha}(\xi)=0$ for a.e. $\xi$ and all $\alpha \in \bigcup_{j \in \mathbb{Z}} B^{j} \mathbb{Z}^{n} \backslash\{0\}$. If $\alpha \in \Lambda^{*} \backslash \bigcup_{j \in \mathbb{Z}} B^{j} \mathbb{Z}^{n}$, then obviously $\tilde{t}_{\alpha}=0$; hence equation (4.14) holds for $\alpha \in \Lambda^{*}$. This shows that the mixed dual Gramian $\widetilde{G}(\xi)$ is the identity operator on $\ell^{2}\left(\Lambda^{*}\right)$ for a.e. $\xi \in I_{\Lambda_{0}^{*}}$, which is equivalent to assertion (iii).

The last implication (iii) $\Rightarrow$ (ii) is obvious.

It is possible to give an alternative proof of Theorem 4.2 using the ideas of polarization from the proof of Theorem 4.1. Since the equivalence (i) $\Leftrightarrow$ (iv) in Theorem 4.2 is well known, we will only (re)prove (ii) $\Leftrightarrow$ (iii) $\Leftrightarrow$ (iv) here.

Another proof of Theorem 4.2. Let $\Lambda \subset \mathbb{Z}^{n}$. For $f \in \mathscr{D}$, we define the $\Lambda$-periodic function $\tilde{w}_{\Lambda}^{q}(x)$ by

$$
\tilde{w}_{\Lambda}^{q}(x)=N_{\Lambda}^{q}\left(T_{x} f, \Psi, \Phi\right)=\sum_{l=1}^{L} \sum_{j \in \mathbb{Z}} \sum_{k \in \mathrm{K}_{j}}\left\langle T_{x} f, d_{j} T_{k} D_{A^{j}} \psi_{l}\right\rangle\left\langle d_{j} T_{k} D_{A^{j}} \phi_{l}, T_{x} f\right\rangle,
$$

where $d_{j}=\#\left\{\Lambda /\left(\Lambda \cap A^{-j} \mathbb{Z}^{n}\right)\right\}^{-1 / 2}$ and $\mathrm{K}_{j}$ is given by (3.3). The series in (4.15) converges absolutely since $\mathcal{A}_{\Lambda}^{q}(\Psi)$ and $\mathcal{A}_{\Lambda}^{q}(\Phi)$ are Bessel sequences. Applying polarization identities as in the proof of Theorem 4.1 yields

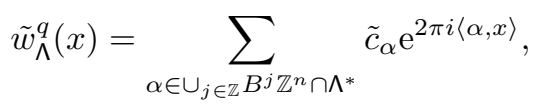

where the coefficients $\left\{\tilde{c}_{\alpha}\right\}$ are given in (4.11).

Assume (ii) holds. It is well known that under the Bessel condition the weak duality of frames is equivalent to the duality of frames; see for example [7, Theorem 5.6.2]. Hence, (ii) is equivalent to $N_{\Lambda_{0}}^{q}(f, \Psi, \Phi)=\|f\|^{2}$ for all $f \in L^{2}\left(\mathbb{R}^{n}\right)$. Since $\left\|T_{x} f\right\|=\|f\|$, this implies that $\tilde{w}_{\Lambda_{0}}^{q}(x)=\|f\|^{2}$. By uniqueness of coefficients of the Fourier series of $\tilde{w}_{\Lambda_{0}}^{q}$, this happens only when

$$
\tilde{c}_{\alpha}=\|f\|^{2} \delta_{\alpha, 0} \quad \text { for } \alpha \in \bigcup_{j \in \mathbb{Z}} B^{j} \mathbb{Z}^{n} \cap \Lambda^{*} .
$$

Following the proof of Theorem 4.1 we immediately have that this implies $\tilde{t}_{\alpha}=\delta_{\alpha, 0}$ almost everywhere for $\alpha \in \bigcup_{j \in \mathbb{Z}} B^{j} \mathbb{Z}^{n} \cap \Lambda^{*}$. In particular, since $\mathbb{Z}^{n} \subset \Lambda^{*}$, we have $\tilde{t}_{\alpha}(\xi)=\delta_{\alpha, 0}$ for a.e. $\xi$ and $\alpha \in \mathbb{Z}^{n}$. This is precisely assertion (iv). 
Assume (iv) holds. By a change of variables, this implies that $\tilde{t}_{\alpha}(\xi)=\delta_{\alpha, 0}$ for a.e. $\xi$ and all $\alpha \in \bigcup_{j \in \mathbb{Z}} B^{j} \mathbb{Z}^{n}$. Therefore,

$$
\tilde{c}_{\alpha}=\|f\|^{2} \delta_{\alpha, 0} \quad \text { for } \alpha \in \bigcup_{j \in \mathbb{Z}} B^{j} \mathbb{Z}^{n},
$$

and we note that these equations are independent of $\Lambda$. Hence, by (4.16), for any $\Lambda \subset \mathbb{Z}^{n}$,

$$
N_{\Lambda}^{q}(f, \Psi, \Phi)=\tilde{w}_{\Lambda}^{q}(0)=\tilde{c}_{0}=\|f\|^{2} \quad \text { for all } f \in \mathscr{D} .
$$

By a density argument, this equality holds for all $f \in L^{2}\left(\mathbb{R}^{n}\right)$, and assertion (iii) follows.

Remark 6. It is apparent from the proof above that the equivalence of (ii), (iii), and (iv) in Theorem 4.2 holds under the weaker assumption that $\mathcal{A}_{\Lambda_{0}}^{q}(\Psi)$ and $\mathcal{A}_{\Lambda_{0}}^{q}(\Phi)$ are Bessel sequences in $L^{2}\left(\mathbb{R}^{n}\right)$ for some $\Lambda_{0} \subset \mathbb{Z}^{n}$.

\section{Diagonal affine Systems}

In this section we study a particularly interesting subclass of generators where the equivalence between affine and quasi-affine frames exhibits the largest degree of symmetry. This is a class of diagonal affine systems for which the off-diagonal functions $t_{\alpha}$ defined below vanish. We show that the class of diagonal affine frames consists precisely of quasi-affine frames having a canonical dual quasi-affine frame. This extends a result of Weber and the first author 5 from the setting of integer dilations to that of rational dilations.

Definition 5.1. For a given dilation matrix $A$ and $\Psi \subset L^{2}\left(\mathbb{R}^{n}\right)$ we introduce the family of functions $\left\{t_{\alpha}\right\}_{\alpha \in \mathbb{Z}^{n}}$ on $\mathbb{R}^{n}$ by

$$
t_{\alpha}(\xi)=\sum_{\psi \in \Psi} \sum_{j \in \mathbb{Z}: \alpha \in B^{j} \mathbb{Z}^{n}} \hat{\psi}\left(B^{-j} \xi\right) \overline{\hat{\psi}\left(B^{-j}(\xi+\alpha)\right)} \quad \text { for } \xi \in \mathbb{R}^{n} .
$$

In particular,

$$
t_{0}(\xi)=\sum_{\psi \in \Psi} \sum_{j \in \mathbb{Z}}\left|\hat{\psi}\left(B^{j} \xi\right)\right|^{2} .
$$

We say that the affine system $\mathcal{A}(\Psi)$ is diagonal if $t_{\alpha}(\xi)=0$ a.e. for all $\alpha \in \mathbb{Z}^{n} \backslash\{0\}$.

Note that the series in (5.1) converges absolutely for a.e. $\xi$ in light of Remark 5 , In addition, if $\Psi \subset L^{2}\left(\mathbb{R}^{n}\right)$ generates an affine Bessel sequence $\mathcal{A}(\Psi)$ with bound $C_{2}$, or a quasi-affine Bessel sequence $\mathcal{A}_{\Lambda}^{q}(\Psi)$ for some lattice $\Lambda$, then each $t_{\alpha}$ is well defined and essentially bounded in light of Proposition 3.2 and

$$
\begin{aligned}
\sum_{\psi \in \Psi} \sum_{j \in \mathbb{Z}: \alpha \in B^{j} \mathbb{Z}^{n}}\left|\hat{\psi}\left(B^{-j} \xi\right) \overline{\hat{\psi}\left(B^{-j}(\xi+\alpha)\right)}\right| & \leq \frac{1}{2} \sum_{\psi \in \Psi} \sum_{j \in \mathbb{Z}: \alpha \in B^{j} \mathbb{Z}^{n}}\left|\hat{\psi}\left(B^{-j} \xi\right)\right|^{2} \\
& +\frac{1}{2} \sum_{\psi \in \Psi} \sum_{j \in \mathbb{Z}: \alpha \in B^{j} \mathbb{Z}^{n}}\left|\hat{\psi}\left(B^{-j}(\xi+\alpha)\right)\right|^{2} \leq C_{2} .
\end{aligned}
$$

Now, with the extra assumption $t_{\alpha}(\xi)=0$ a.e. for $\alpha \in \mathbb{Z}^{n} \backslash\{0\}$, we have the following equivalence result. 
Theorem 5.1. Let $A \in G L_{n}(\mathbb{Q})$ be expansive, $\Psi \subset L^{2}\left(\mathbb{R}^{n}\right)$ and let $C_{1}, C_{2}>0$ be constants. Suppose that the affine system $\mathcal{A}(\Psi)$ is diagonal. Then the following assertions are equivalent:

(i) the affine system $\mathcal{A}(\Psi)$ is a frame for $L^{2}\left(\mathbb{R}^{n}\right)$ with bounds $C_{1}, C_{2}$;

(ii) the quasi-affine system $\mathcal{A}_{\Lambda_{0}}^{q}(\Psi)$ is a frame for $L^{2}\left(\mathbb{R}^{n}\right)$ with bounds $C_{1}, C_{2}$ for some integer lattice $\Lambda_{0} \subset \mathbb{Z}^{n}$;

(iii) the quasi-affine system $\mathcal{A}_{\Lambda}^{q}(\Psi)$ is a frame for $L^{2}\left(\mathbb{R}^{n}\right)$ with bounds $C_{1}, C_{2}$ for all integer lattices $\Lambda \subset \mathbb{Z}^{n}$;

$$
C_{1} \leq \sum_{\psi \in \Psi} \sum_{j \in \mathbb{Z}}\left|\hat{\psi}\left(B^{j} \xi\right)\right|^{2} \leq C_{2} \quad \text { for a.e. } \xi \in \mathbb{R}^{n} .
$$

Proof. Let $\Lambda \subset \mathbb{Z}^{n}$ be a lattice in $\mathbb{R}^{n}$. For fixed $f \in \mathscr{D}$, let $w$ and $w_{\Lambda}^{q}$ be the functions introduced in (3.13) and (3.17). By a change of summation order, using absolute convergence of the series, these functions can be written as

$$
w(x)=\sum_{\alpha \in \bigcup_{j \in \mathbb{Z}} B^{j} \mathbb{Z}^{n}} c_{\alpha} \mathrm{e}^{2 \pi i\langle\alpha, x\rangle}, \quad w_{\Lambda}^{q}(x)=\sum_{\alpha \in \bigcup_{j \in \mathbb{Z}} B^{j} \mathbb{Z}^{n} \cap \Lambda^{*}} c_{\alpha} \mathrm{e}^{2 \pi i\langle\alpha, x\rangle},
$$

where

$$
\begin{aligned}
& c_{\alpha}=\int_{\mathbb{R}^{n}} \hat{f}(\xi) \overline{\hat{f}(\xi+\alpha)} \sum_{\psi \in \Psi} \sum_{j \in \mathbb{Z}: \alpha \in B^{j} \mathbb{Z}^{n}} \overline{\hat{\psi}\left(B^{-j} \xi\right)} \hat{\psi}\left(B^{-j}(\xi+\alpha)\right) \mathrm{d} \xi \\
& =\int_{\mathbb{R}^{n}} \hat{f}(\xi) \overline{\hat{f}(\xi+\alpha) t_{\alpha}(\xi)} \mathrm{d} \xi, \quad \text { for } \alpha \in \bigcup_{j \in \mathbb{Z}} B^{j} \mathbb{Z}^{n} .
\end{aligned}
$$

Our standing assumption in this theorem is that $t_{\alpha}(\xi)=0$ a.e. for $\alpha \in \mathbb{Z}^{n} \backslash\{0\}$. By a change of variables, this implies $t_{\alpha}(\xi)=0$ a.e. for $\alpha \in \bigcup_{j \in \mathbb{Z}} B^{j} \mathbb{Z}^{n} \backslash\{0\}$. Thus the expressions in (5.2) reduce to

$$
w(x)=w_{\Lambda}^{q}(x)=c_{0}=\int_{\mathbb{R}^{n}}|\hat{f}(\xi)|^{2} t_{0}(\xi) \mathrm{d} \xi \quad \text { for all } x \in \mathbb{R}^{n} ;
$$

hence $w$ and $w_{\wedge}^{q}$ are equal and constant functions of $x$. Therefore

$$
N(f)=w(0)=w_{\Lambda}^{q}(0)=N_{\Lambda}^{q}(f)
$$

for $f \in \mathscr{D}$. Since $\mathscr{D}$ is dense in $L^{2}\left(\mathbb{R}^{n}\right)$, we find that (i) $\Leftrightarrow$ (ii) $\Leftrightarrow$ (iii). Note that (i) $\Rightarrow$ (iii) also follows directly from Theorem 3.5 .

We will verify that (i) $\Leftrightarrow$ (iv). In terms of the $t_{\alpha}$-functions, assertion (iv) reads as $C_{1} \leq t_{0}(\xi) \leq C_{2}$ almost everywhere. By the above and an application of the Plancherel theorem, assertion (i) is equivalent to

$$
C_{1}\langle\hat{f}, \hat{f}\rangle \leq\left\langle t_{0} \hat{f}, \hat{f}\right\rangle \leq C_{2}\langle\hat{f}, \hat{f}\rangle \quad \text { for } f \in L^{2}\left(\mathbb{R}^{n}\right)
$$

This implies that

$$
C_{1} \leq t_{0}(\xi) \leq C_{2} \quad \text { for a.e. } \xi \in \mathbb{R}^{n},
$$

which, on the other hand, clearly implies (5.4).

As a corollary we have the following converse of Theorem 3.5

Corollary 5.2. Let $A \in G L_{n}(\mathbb{Q})$ be expansive, $\Psi \subset L^{2}\left(\mathbb{R}^{n}\right)$, and let $\mathcal{A}(\Psi)$ be diagonal. Suppose that the $\Lambda_{0}$-oversampled quasi-affine system $\mathcal{A}_{\Lambda_{0}}^{q}(\Psi)$ is a frame for $L^{2}\left(\mathbb{R}^{n}\right)$ with bounds $C_{1}, C_{2}$ for some integer lattice $\Lambda_{0} \subset \mathbb{Z}^{n}$. Then, the affine system $\mathcal{A}(\Psi)$ is a frame for $L^{2}\left(\mathbb{R}^{n}\right)$ with bounds $C_{1}, C_{2}$. 
As a direct consequence of Theorem 3.5 and Corollary 5.2 we generalize the equivalence of affine and quasi-affine Parseval frames due to the first author [3, Theorem 3.4]; see also [14, Theorem 2.17].

Theorem 5.3. Suppose $A \in G L_{n}(\mathbb{Q})$ is expansive and $\Psi \subset L^{2}\left(\mathbb{R}^{n}\right)$. Then the following assertions are equivalent:

(i) the affine system $\mathcal{A}(\Psi)$ is a Parseval frame for $L^{2}\left(\mathbb{R}^{n}\right)$;

(ii) the quasi-affine system $\mathcal{A}_{\Lambda_{0}}^{q}(\Psi)$ is a Parseval frame for $L^{2}\left(\mathbb{R}^{n}\right)$ for some integer lattice $\Lambda_{0} \subset \mathbb{Z}^{n}$;

(iii) the quasi-affine system $\mathcal{A}_{\Lambda}^{q}(\Psi)$ is a Parseval frame for $L^{2}\left(\mathbb{R}^{n}\right)$ for all integer lattices $\Lambda \subset \mathbb{Z}^{n}$.

Proof. The implication (i) $\Rightarrow$ (iii) is a special case of Theorem 3.5, and (iii) $\Rightarrow$ (ii) is obvious. Proposition 3.2 and the proof of Proposition 3.3 show that the local integrability condition (3.16) for the quasi-affine system is satisfied; hence we can apply Theorem 2.4 to $\mathcal{A}_{\Lambda}^{q}(\Psi)$. By equations (2.14), (3.10) and (3.11) this implies that $t_{\alpha}=0$ for $\alpha \in \mathbb{Z}^{n} \backslash\{0\}$; hence the affine system is diagonal. An application of Corollary 5.2 gives us (ii) $\Rightarrow$ (i).

5.1. Canonical dual quasi-affine frames. Our next aim is to characterize when the canonical dual of a quasi-affine frame is also a quasi-affine frame. To achieve this we need the following result resembling [5, Proposition 1].

Theorem 5.4. Let $A \in G L_{n}(\mathbb{Q})$ be expansive. Suppose the $\mathcal{A}_{\Lambda_{0}}^{q}(\Psi)$ is a frame for some $\Lambda_{0} \subset \mathbb{Z}^{n}$, which has a dual quasi-affine frame $\mathcal{A}_{\Lambda_{0}}^{q}(\Phi)$. Then, for any $S \in B\left(L^{2}\left(\mathbb{R}^{n}\right)\right)$ we have

$$
S \in \mathcal{C}_{\psi}\left(\mathcal{A}_{\Lambda_{0}}^{q}\right) \text { for all } \psi \in \Psi \quad \Leftrightarrow \quad S \in\left\{D_{A}, T_{\lambda}: \lambda \in \Lambda_{0}\right\}^{\prime} .
$$

Note that we need to assume a much stronger hypothesis than the assumption of [5. Proposition 1], saying that the quasi-affine system $\mathcal{A}_{\mathbb{Z}^{n}}^{q}(\Psi)$ is complete in $L^{2}\left(\mathbb{R}^{n}\right)$.

Proof. The fact that $\mathcal{A}_{\Lambda_{0}}^{q}(\Psi)$ and $\mathcal{A}_{\Lambda_{0}}^{q}(\Phi)$ are dual frames implies that the fundamental equations (4.2) and (4.3) hold; see Remark 6. By Theorem 4.1, the affine system $\mathcal{A}(\Psi)$ is complete in $L^{2}\left(\mathbb{R}^{n}\right)$.

Suppose that $S \in \mathcal{C}_{\psi}\left(\mathcal{A}_{\Lambda_{0}}^{q}\right)$. Since the quasi-affine system $\mathcal{A}_{\Lambda_{0}}^{q}(\Psi)$ is $\Lambda_{0^{-}}$SI, $S$ must commute with translations $T_{\lambda}, \lambda \in \Lambda_{0}$. Likewise, since the affine system $\mathcal{A}(\Psi)$ is a part of the quasi-affine system $\mathcal{A}_{\Lambda_{0}}^{q}(\Psi)$ (up to normalizing constants), $S \in \mathcal{C}_{\psi}(\mathcal{A})$. Since the affine system $\mathcal{A}(\Psi)$ is complete in $L^{2}\left(\mathbb{R}^{n}\right)$ and $\mathcal{A}(\Psi)$ is dilation-invariant, $S$ must commute with the dilation operator $D_{A}$.

Conversely, if $S \in\left\{D_{A}, T_{\lambda}: \lambda \in \Lambda_{0}\right\}^{\prime}$, then clearly $S$ belongs to the local commutant $\mathcal{C}_{\psi}\left(\mathcal{A}_{\Lambda_{0}}^{q}\right)$ for any choice of $\psi \in L^{2}\left(\mathbb{R}^{n}\right)$.

Remark 7. Note that if $S \in\left\{D_{A}, T_{\lambda}: \lambda \in \Lambda_{0}\right\}^{\prime}$, then $S$ commutes with all translation $T_{\lambda}, \lambda \in \mathbb{R}^{n}$. Indeed, by $T_{A^{j} \lambda}=D_{A^{-j}} T_{\lambda} D_{A^{j}}, S$ must commute with $T_{A^{j} \lambda}$ for $j \in \mathbb{Z}$ and $\lambda \in \Lambda_{0}$. Since $A$ is expansive, $\bigcup_{j \in \mathbb{Z}} A^{j} \Lambda_{0}$ is dense in $\mathbb{R}^{n}$. Hence, by continuity of $x \mapsto T_{x} f$ for $f \in L^{2}\left(\mathbb{R}^{n}\right)$, we have $S \in\left\{D_{A}, T_{\lambda}: \lambda \in \mathbb{R}^{n}\right\}^{\prime}$. In fact, we have the following lemma which is a straightforward generalization of [5, Lemma $2]$. 
Lemma 5.5. Let $A \in G L_{n}(\mathbb{R})$ be expansive, $\Lambda$ a lattice, and $S \in B\left(L^{2}\left(\mathbb{R}^{n}\right)\right)$. Then, $S \in\left\{D_{A}, T_{\lambda}: \lambda \in \Lambda\right\}^{\prime}$ if, and only if, $S$ is a $B$-dilation periodic Fourier multiplier, i.e., there exists a function $s \in L^{\infty}\left(\mathbb{R}^{n}\right)$ such that

$$
\widehat{S f}(\xi)=s(\xi) \hat{f}(\xi) \text { for a.e. } \xi,
$$

where $s(\xi)=s(B \xi)$ for a.e. $\xi$.

Proof. Assume $S \in\left\{D_{A}, T_{\lambda}: \lambda \in \Lambda\right\}^{\prime}$. By $T_{A^{j} \lambda}=D_{A^{-j}} T_{\lambda} D_{A^{j}}, S$ commutes with $T_{A^{j} \lambda}$ for $j \in \mathbb{Z}$ and $\lambda \in \Lambda$, i.e.,

$$
S T_{k}=T_{k} S \quad \text { for } k \in \bigcup_{j \in \mathbb{Z}} A^{j} \Lambda .
$$

The union $\bigcup_{j \in \mathbb{Z}} A^{j} \Lambda$ is dense in $\mathbb{R}^{n}$ since $A$ is expansive. For $x \in \mathbb{R}^{n}$ take $\left\{k_{n}\right\}_{n \in \mathbb{N}}$ from $\bigcup_{j \in \mathbb{Z}} A^{j} \Lambda$ such that $k_{n} \rightarrow x$. By continuity of $x \mapsto T_{x} f$ for $f \in L^{2}\left(\mathbb{R}^{n}\right)$, $k_{n} \rightarrow x$ implies $T_{k_{n}} f \rightarrow T_{x} f$ in the $L^{2}$ norm, i.e., $T_{k_{n}} \rightarrow T_{x}$ in the strong operator topology. Hence, by equation (5.5), we have $S T_{x}=T_{x} S$, proving that $S$ is a Fourier multiplier. Finally, by $D_{A} S=S D_{A}$,

$$
\mathcal{F} D_{A} S f(\xi)=\int_{\mathbb{R}^{n}} D_{A} S f(x) \mathrm{e}^{-2 \pi i x \cdot \xi} \mathrm{d} x=|\operatorname{det} A|^{-1 / 2} s\left(B^{-1} \xi\right) \hat{f}\left(B^{-1} \xi\right),
$$

and

$$
\mathcal{F} S D_{A} f(\xi)=s(\xi) \int_{\mathbb{R}^{n}} D_{A} f(x) \mathrm{e}^{-2 \pi i x \cdot \xi} \mathrm{d} x=|\operatorname{det} A|^{-1 / 2} s(\xi) \hat{f}\left(B^{-1} \xi\right),
$$

we have $B$-periodicity of the symbol $s$.

Conversely, assume $S$ is a Fourier multiplier with a $B$-dilation periodic symbol. The operator $S$ commutes with all translations by the Fourier multiplier property and with dilations $D_{A}$ by the $B$-dilation periodicity of the symbol and the two displayed equations above.

Theorem 5.6. Let $A \in G L_{n}(\mathbb{Q})$ be expansive. Suppose the oversampled quasiaffine system $\mathcal{A}_{\Lambda_{0}}^{q}(\Psi)$ is a frame for $L^{2}\left(\mathbb{R}^{n}\right)$ for some integer lattices $\Lambda_{0} \subset \mathbb{Z}^{n}$. Then the canonical dual frame of $\mathcal{A}_{\Lambda_{0}}^{q}(\Psi)$ has the form $\mathcal{A}_{\Lambda_{0}}^{q}(\Phi)$ for some set of functions $\Phi \subset L^{2}\left(\mathbb{R}^{n}\right)$ with cardinality $\# \Phi=\# \Psi$ if, and only if,

$$
t_{\alpha}(\xi)=\sum_{\psi \in \Psi} \sum_{j \in \mathbb{Z}: \alpha \in B^{j} \mathbb{Z}^{n}} \hat{\psi}\left(B^{-j} \xi\right) \overline{\hat{\psi}\left(B^{-j}(\xi+\alpha)\right)}=0 \quad \text { for all } \alpha \in \mathbb{Z}^{n} \backslash\{0\} .
$$

Moreover, in the positive case $\mathcal{A}_{\Lambda}^{q}(\Psi)$ is a frame for all integer lattices $\Lambda \subset \mathbb{Z}^{n}$ and its canonical dual frame is $\mathcal{A}_{\Lambda}^{q}(\Phi)$.

Proof. Let $S_{\Lambda_{0}}^{q}$ be the frame operator of the quasi-affine system $\mathcal{A}_{\Lambda_{0}}^{q}(\Psi)$. Since $\mathcal{A}_{\Lambda_{0}}^{q}(\Psi)$ is a frame, equation (3.12) is satisfied. Hence the expression for $w_{\Lambda_{0}}^{q}$ in (5.2) holds for $f \in \mathscr{D}$.

Assume that the canonical dual of $\mathcal{A}_{\Lambda_{0}}^{q}(\Psi)$ has the form $\mathcal{A}_{\Lambda_{0}}^{q}(\Phi)$, i.e., $S_{\Lambda_{0}}^{q} \in$ $\mathcal{C}_{\psi}\left(\mathcal{A}_{\Lambda_{0}}^{q}\right)$ for all $\psi \in \Psi$. By Theorem [5.4 and Remark 7, $S_{\Lambda_{0}}^{q} \in\left\{D_{A}, T_{\lambda}: \lambda \in \mathbb{R}^{n}\right\}^{\prime}$. Hence

$$
w_{\Lambda_{0}}^{q}(x)=\left\langle S_{\Lambda_{0}}^{q} T_{x} f, T_{x} f\right\rangle=\left\langle T_{x} S_{\Lambda_{0}}^{q} f, T_{x} f\right\rangle=\left\langle S_{\Lambda_{0}}^{q} f, f\right\rangle \quad \forall x \in \mathbb{R}^{n},
$$

which shows that $w_{\Lambda_{0}}^{q}$ is constant for every $f \in \mathscr{D}$. 
For each $f \in \mathscr{D}$ we express $w_{\Lambda_{0}}^{q}$ as the $\Lambda_{0}$-periodic Fourier series (5.2). Such a Fourier series is identically constant if, and only if,

$$
c_{\alpha} \equiv \int_{\mathbb{R}^{n}} \hat{f}(\xi) \bar{f}(\xi+\alpha) t_{\alpha}(\xi) \mathrm{d} \xi=0 \quad \text { for all } \alpha \in\left(\bigcup_{j \in \mathbb{Z}} B^{j} \mathbb{Z}^{n} \cap \Lambda_{0}^{*}\right) \backslash\{0\},
$$

by the uniqueness of the Fourier coefficients. In particular, this equality holds for $\alpha \in \mathbb{Z}^{n} \backslash\{0\}$ since $\mathbb{Z}^{n} \subset \Lambda_{0}^{*}$. Fix $\alpha \in \mathbb{Z}^{n} \backslash\{0\}$. Let $I_{\Lambda_{0}^{*}}$ denote a fundamental domain of $\Lambda_{0}^{*}$ and, for $l \in \Lambda_{0}^{*}$, let $I_{l}=I_{\Lambda_{0}^{*}}+l$. Define $f$ by

$$
\hat{f}(\xi):= \begin{cases}\frac{1}{t_{\alpha}(\xi)} & \text { for } \xi \in I_{l}, \\ 0 & \text { otherwise. }\end{cases}
$$

Since $t_{\alpha}$ is bounded by the Bessel bound $C_{2}$, we have $f \in \mathscr{D}$. Now,

$$
0=\int_{\mathbb{R}^{n}} \hat{f}(\xi) \overline{\hat{f}(\xi+\alpha) t_{\alpha}(\xi)} \mathrm{d} \xi=\int_{I_{l}} t_{\alpha}(\xi) \overline{t_{\alpha}(\xi)} \mathrm{d} \xi=\int_{I_{l}}\left|t_{\alpha}(\xi)\right|^{2} \mathrm{~d} \xi
$$

for each $l \in \Lambda_{0}^{*}$. Since $\bigcup_{l \in \Lambda_{0}^{*}} I_{l}=\mathbb{R}^{n}$ we deduce that $t_{\alpha}(\xi)=0$ for a.e. $\xi \in \mathbb{R}^{n}$, and the theorem is half proved.

Conversely, assume $t_{\alpha}(\xi)=0$ for $\alpha \in \mathbb{Z}^{n} \backslash\{0\}$. Then $t_{\alpha}(\xi)=0$ for $\alpha \in$ $\left(\bigcup_{j \in \mathbb{Z}} B^{j} \mathbb{Z}^{n}\right) \backslash\{0\}$ by a change of variables. In particular, $t_{\alpha}(\xi)=0$ for $\alpha \in$ $\left(\bigcup_{j \in \mathbb{Z}} B^{j} \mathbb{Z}^{n} \cap \Lambda_{0}^{*}\right) \backslash\{0\}$. Hence $w_{\Lambda_{0}}^{q}(x)=c_{0}$ for every $x \in \mathbb{R}^{n}$, i.e., $w_{\Lambda_{0}}^{q}$ is constant on $\mathbb{R}^{n}$ for every $f \in \mathscr{D}$. Therefore, for every $x \in \mathbb{R}^{n}$,

$$
\left\langle S_{\Lambda_{0}}^{q} T_{x} f, T_{x} f\right\rangle=w_{\Lambda_{0}}^{q}(x)=w_{\Lambda_{0}}^{q}(0)=\left\langle S_{\Lambda_{0}}^{q} f, f\right\rangle \quad \text { for } f \in \mathscr{D} .
$$

This equality extends to all $f \in L^{2}\left(\mathbb{R}^{n}\right)$ by a density argument; hence

$$
\left\langle\left(T_{-x} S_{\Lambda_{0}}^{q} T_{x}-S_{\Lambda_{0}}^{q}\right) f, f\right\rangle=0 \quad \text { for } f \in L^{2}\left(\mathbb{R}^{n}\right) .
$$

We conclude that $S_{\Lambda_{0}}^{q} T_{x}=T_{x} S_{\Lambda_{0}}^{q}$ for all $x \in \mathbb{R}^{n}$, in other words, $S_{\Lambda_{0}}^{q}$ is a Fourier multiplier:

$$
\widehat{S_{\Lambda_{0}}^{q} f}(\xi)=s(\xi) \hat{f}(\xi) \quad \text { for a.e. } \xi \in \mathbb{R}^{n} \text { and all } f \in L^{2}\left(\mathbb{R}^{n}\right)
$$

for some symbol $s \in L^{\infty}\left(\mathbb{R}^{n}\right)$. We claim the symbol of $S_{\Lambda_{0}}^{q}$ is

$$
s(\xi)=t_{0}(\xi)=\sum_{\psi \in \Psi} \sum_{j \in \mathbb{Z}}\left|\hat{\psi}\left(B^{j} \xi\right)\right|^{2} .
$$

This function is obviously a $B$-dilation periodic function; that is, $s(\xi)=s(B \xi)$. By Proposition 3.2 the function is bounded by the upper frame bound $s(\xi) \leq C_{2}$ for a.e. $\xi$, so $s \in L^{\infty}\left(\mathbb{R}^{n}\right)$. By the Plancherel theorem, we see

$$
w_{\Lambda_{0}}^{q}(0)=\left\langle S_{\Lambda_{0}}^{q} f, f\right\rangle=\left\langle\widehat{S_{\Lambda_{0}}^{q} f}, \hat{f}\right\rangle \quad \text { for all } f \in \mathscr{D},
$$

and, by (5.3) with $\alpha=0$, that

$$
c_{0}=\int_{\mathbb{R}^{n}} \hat{f}(\xi) \overline{\hat{f}(\xi)} \sum_{\psi \in \Psi} \sum_{j \in \mathbb{Z}}\left|\hat{\psi}\left(B^{j} \xi\right)\right|^{2} \mathrm{~d} \xi .
$$

Since $w_{\Lambda_{0}}^{q}(x)=c_{0}$ for all $x \in \mathbb{R}^{n}$, we have, in particular,

$$
\left\langle\widehat{S_{\Lambda_{0}}^{q} f}, \hat{f}\right\rangle=w_{\Lambda_{0}}^{q}(0)=c_{0}=\langle s \hat{f}, \hat{f}\rangle \quad \text { for all } f \in \mathscr{D} \text {. }
$$


Therefore, $s$ is a $B$-dilation periodic symbol of $S_{\Lambda_{0}}^{q}$ implying that $S_{\Lambda_{0}}^{q}$ commutes with $D_{A}$; see Lemma 5.5. The frame operator $S_{\Lambda_{0}}^{q}$ therefore belongs to $\left\{D_{A}, T_{\lambda}: \lambda \in \Lambda_{0}\right\}^{\prime}$. As a result we find that $\left(S_{\Lambda_{0}}^{q}\right)^{-1} \in \mathcal{C}_{\psi}\left(\mathcal{A}_{\Lambda_{0}}^{q}\right)$ for $\psi \in \Psi$. This is equivalent to the canonical dual of $\mathcal{A}_{\Lambda_{0}}^{q}(\Psi)$ having the quasi-affine structure with the same number of generators.

\section{Broken Symmetry BetWeEn the Integer and RATIONAL CASE}

The goal of this section is to illustrate fundamental differences between integer and rational cases. That is, a mere fact that a quasi-affine system is a frame does not imply that an affine system must be a frame as well. This kind of phenomenon cannot happen for integer dilations where we have a perfect equivalence of the frame property between affine and quasi-affine systems. Moreover, this cannot happen for Parseval frames due to Theorem 5.3 or, more generally, for affine frames having duals by Theorem 4.2. Moreover, Theorem 6.1 shows the optimality of our results. That is, the assumption of uniformity of frame bounds of quasi-affine systems in Theorem 3.8 cannot be removed in general.

Theorem 6.1. Let $1<a \in \mathbb{Q} \backslash \mathbb{Z}$ be a rational non-integer dilation factor. Then there exists a function $\psi \in L^{2}(\mathbb{R})$ such that $\mathcal{A}_{\Lambda}^{q}(\psi)$ is a frame for any oversampling lattice $\Lambda \subset \mathbb{Z}$, but yet $\mathcal{A}(\psi)$ is not a frame.

Remark 8. In light of Theorem 3.8, the frame bounds of the quasi-affine systems $\mathcal{A}_{\Lambda}^{q}(\psi)$ are not uniform for all lattices $\Lambda \subset \mathbb{Z}$. In fact, we will see that the lower frame bound of $\mathcal{A}_{\Lambda}^{q}(\psi)$ drops to 0 as a lattice $\Lambda$ gets sparser and sparser. Consequently, in the limiting case, when no oversampling is present, we obtain an affine system $\mathcal{A}(\psi)$ which is not a frame due to the failure of the lower frame bound.

We will need the following well-known result; see [16, Theorem 13.0.1] or the proof of [10, Lemma 3.4].

Theorem 6.2. Suppose that $\psi \in L^{2}(\mathbb{R})$ is such that $\hat{\psi} \in L^{\infty}(\mathbb{R})$ and

$$
\begin{aligned}
& \hat{\psi}(\xi)=O\left(|\xi|^{\delta}\right) \quad \text { as } \xi \rightarrow 0, \\
& \hat{\psi}(\xi)=O\left(|\xi|^{-1 / 2-\delta}\right) \quad \text { as }|\xi| \rightarrow \infty,
\end{aligned}
$$

for some $\delta>0$. Then the affine system $\mathcal{A}(\psi)$ is a Bessel sequence.

We define the space $\check{L}^{2}(K)$, invariant under all translations, by

$$
\check{L}^{2}(K)=\left\{f \in L^{2}(\mathbb{R}): \operatorname{supp} \hat{f} \subset K\right\}
$$

for measurable subsets $K$ of $\mathbb{R}$.

Proof of Theorem 6.1. Choose $\delta>0$ so that $\frac{1}{a(a+1)}<\delta<\frac{1}{2 a}<\frac{1}{a^{2}+1}$. Define $\psi \in L^{2}(\mathbb{R})$ as $\hat{\psi}=\mathbf{1}_{\left(-a^{2} \delta,-\delta\right) \cup\left(\delta, a^{2} \delta\right)}$. First, we shall show that the affine system $\mathcal{A}(\psi)$ is not a frame. To achieve this we will follow the idea from [5, Example 2]. We will need the following standard identity, which can be shown by the periodization argument

$$
\sum_{k \in \mathbb{Z}}\left|\left\langle f, T_{k} \psi\right\rangle\right|^{2}=\int_{0}^{1}\left|\sum_{k \in \mathbb{Z}} \hat{f}(\xi+k) \overline{\hat{\psi}(\xi+k)}\right|^{2} \mathrm{~d} \xi \quad \text { for any } f \in L^{2}(\mathbb{R}) .
$$


Let $K_{\delta}=\left(1-a^{2} \delta, a^{2} \delta\right)$. By the restriction on $\delta$, we have

$$
K_{\delta} \subset\left(\delta, a^{2} \delta\right) \subset(\delta, 1-\delta) \quad \text { and } \quad K_{\delta}-1 \subset\left(-a^{2} \delta,-\delta\right) \subset(-1+\delta,-\delta) .
$$

Hence, by a direct calculation using (6.1) we have for any $f \in L^{2}(\mathbb{R})$,

$$
\sum_{k \in \mathbb{Z}}\left|\left\langle f, T_{k} \psi\right\rangle\right|^{2}=\int_{K_{\delta}}|\hat{f}(\xi-1)+\hat{f}(\xi)|^{2} \mathrm{~d} \xi+\int_{\left(\delta, 1-a^{2} \delta\right)}|\hat{f}(\xi)|^{2} \mathrm{~d} \xi+\int_{\left(a^{2} \delta, 1-\delta\right)}|\hat{f}(\xi-1)|^{2} \mathrm{~d} \xi
$$

In particular, by restricting (6.2) to a subspace $\check{L}^{2}\left(L_{\delta}\right)$, where

$$
L_{\delta}=(-\infty,-1+\delta) \cup\left(K_{\delta}-1\right) \cup(-\delta, \delta) \cup K_{\delta} \cup(1-\delta, \infty),
$$

we obtain a convenient formula

$$
\sum_{k \in \mathbb{Z}}\left|\left\langle f, T_{k} \psi\right\rangle\right|^{2}=\int_{K_{\delta}}|\hat{f}(\xi-1)+\hat{f}(\xi)|^{2} \mathrm{~d} \xi \quad \text { for any } f \in \check{L}^{2}\left(L_{\delta}\right) .
$$

For any natural number $N$ and sufficiently small $\varepsilon=\varepsilon(N)>0$, we define a function $f_{N} \in L^{2}(\mathbb{R})$ by

$$
\hat{f}_{N}=\sum_{k=0}^{N}\left(\mathbf{1}_{I_{k}^{+}}-\mathbf{1}_{I_{k}^{-}}\right)
$$

where

$$
I_{k}^{+}=\left(\frac{a^{-k}}{a+1}-\varepsilon, \frac{a^{-k}}{a+1}\right), I_{k}^{-}=\left(-\frac{a^{-k}}{a+1}-\varepsilon,-\frac{a^{-k}}{a+1}\right) .
$$

Intuitively, one might think of $\hat{f}_{N}$ as a linear combination of point masses

$$
\varepsilon \sum_{k=0}^{N}\left(\delta_{a^{-k} /(a+1)}-\delta_{-a^{-k} /(a+1)}\right) .
$$

We claim that

$$
D_{a^{j}} f_{N} \in \check{L}^{2}\left(L_{\delta}\right) \quad \text { for all } j \in \mathbb{Z} .
$$

Indeed, (6.6) follows immediately from

$$
a^{j}\left(I_{k}^{+} \cup I_{k}^{-}\right) \subset \begin{cases}(-\delta, \delta), & j \leq k-1, \\ \left(K_{\delta}-1\right) \cup K_{\delta}, & j=k, k+1, \\ (-\infty,-1+\delta) \cup(1-\delta, \infty), & j \geq k+2,\end{cases}
$$

for $k=0, \ldots, N$ and for sufficiently small $\varepsilon=\varepsilon(N)>0$, i.e.,

$$
0<\varepsilon<\min \left\{a^{-N+1}\left(\delta-\frac{1}{a(a+1)}\right), a^{-N-2}\left(\frac{a^{2}}{a+1}-1+\delta\right)\right\} .
$$

Let $S$ be the frame operator corresponding to the affine system $\mathcal{A}(\psi)$. Note that by Theorem 6.2, $S$ is bounded. Our goal is to show that $S$ is not bounded from below. Combining (6.3)- -6.6 we have

$$
\begin{aligned}
\left\|S f_{N}\right\|^{2} & =\sum_{j \in \mathbb{Z}} \sum_{z \in \mathbb{Z}}\left|\left\langle f_{N}, D_{a^{j}} T_{z} \psi\right\rangle\right|^{2}=\sum_{j \in \mathbb{Z}} \sum_{z \in \mathbb{Z}}\left|\left\langle D_{a^{j}} f_{N}, T_{z} \psi\right\rangle\right|^{2} \\
& =\sum_{j=0}^{N+1} a^{-j} \int_{K_{\delta}}\left|\hat{f}_{N}\left(a^{-j}(\xi-1)\right)+\hat{f}_{N}\left(a^{-j} \xi\right)\right|^{2} \mathrm{~d} \xi=4 \varepsilon .
\end{aligned}
$$


Here, we used that for $\xi \in K_{\delta}$

$$
\hat{f}_{N}\left(a^{-j}(\xi-1)\right)+\hat{f}_{N}\left(a^{-j} \xi\right)= \begin{cases}\mathbf{1}_{I_{0}^{+}}(\xi)-\mathbf{1}_{I_{0}^{-}}(\xi-1), & j=0, \\ 0, & j=1, \ldots, N, \\ \mathbf{1}_{a^{N+1} I_{N}^{+}}(\xi)-\mathbf{1}_{a^{N+1} I_{N}^{-}}(\xi-1), & j=N+1 .\end{cases}
$$

The presence of cancellations at scales $j=1, \ldots, N$ is due to translation-dilation linkage of the quadruple of points $\{ \pm a /(a+1), \pm 1 /(a+1)\}$. On the other hand,

$$
\left\|f_{N}\right\|^{2}=\left\|\hat{f}_{N}\right\|^{2}=2 \varepsilon(N+1) .
$$

Since $N$ is arbitrary, this shows that the frame operator $S$ is not bounded from below. Consequently, $\mathcal{A}(\psi)$ is not a frame.

Next, we will show that $\mathcal{A}_{\Lambda}^{q}(\psi)$ is a frame for any choice of lattice $\Lambda \subset \mathbb{Z}$. Since $\mathcal{A}(\psi)$ is a Bessel sequence, Theorem 3.5 yields that $\mathcal{A}_{\Lambda}^{q}(\psi)$ is a Bessel sequence as well. Hence, it remains to establish the lower frame bound for $\mathcal{A}_{\Lambda}^{q}(\psi)$.

Let $a=p / q$, where $p, q \in \mathbb{N}$ are relatively prime, and $l \in \mathbb{N}$ be such that $\Lambda=l \mathbb{Z}$. Let

$$
J_{1}=\max \left\{j \in \mathbb{N}_{0}: p^{j} \text { divides } l\right\}, \quad J_{2}=\max \left\{j \in \mathbb{N}_{0}: q^{j} \text { divides } l\right\} .
$$

Take any $j \in \mathbb{Z}$. Then we have the equality of lattices $a^{-j} \mathbb{Z}+\Lambda=a^{-j} \mathbb{Z} \Leftrightarrow l$ is an integer multiple of $a^{-j}$. Clearly, this is equivalent to $l$ being divisible by $q^{j}$ if $j>0$ or $l$ divisible by $p^{-j}$ if $j<0$. Therefore,

$$
a^{-j} \mathbb{Z}+\Lambda=a^{-j} \mathbb{Z} \quad \Leftrightarrow \quad-J_{1} \leq j \leq J_{2} .
$$

Consequently,

$$
a^{-j} \mathbb{Z}+\Lambda=\frac{1}{c_{j}} a^{-j} \mathbb{Z} \quad \text { for some } c_{j} \geq 2, \text { where } j<-J_{1} \text { or } j>J_{2} .
$$

The properties (6.7) and (6.8) enable us to identify the quasi-affine system $\mathcal{A}_{\Lambda}^{q}(\psi)$. At the scales $-J_{1} \leq j \leq J_{2}$, the quasi-affine system $\mathcal{A}_{\Lambda}^{q}(\psi)$ coincides with the affine system $\mathcal{A}(\psi)$. However, outside of this finite range of scales the quasi-affine system is obtained by oversampling the affine system at a rate $c_{j} \geq 2$. This will lead to a simple form of the frame operator $S_{\Lambda}^{q}$ of the quasi-affine system $\mathcal{A}_{\Lambda}^{q}(\psi)$.

Indeed, suppose that $j\left\langle-J_{1}\right.$ or $j>J_{2}$. By Definition 3.1 and (6.8) the quasiaffine system $\mathcal{A}_{\Lambda}^{q}(\psi)$ at the scale $j$ is

$$
O_{\Lambda}^{a^{-j} \mathbb{Z}}\left(D_{a^{j}} \psi\right)=E^{a^{-j} \mathbb{Z}+\Lambda}\left(\frac{1}{\left|\Lambda /\left(\Lambda \cap a^{-j} \mathbb{Z}\right)\right|^{1 / 2}} D_{a^{j}} \psi\right)=E^{a^{-j} / c_{j} \mathbb{Z}}\left(\left(c_{j}\right)^{-1 / 2} D_{a^{j}} \psi\right) .
$$

Hence,

$$
\begin{aligned}
& \sum_{g \in O_{\Lambda}^{a-j}\left(D_{a^{j}} \psi\right)}|\langle f, g\rangle|^{2}=\frac{1}{c_{j}} \sum_{k \in \mathbb{Z}}\left|\left\langle f, T_{a^{-j} k / c_{j}} D_{a^{j}} \psi\right\rangle\right|^{2} \\
& =\sum_{k \in \mathbb{Z}} \frac{1}{c_{j} a^{j}}\left|\int_{\mathbb{R}} \hat{f}(\xi) \hat{\psi}\left(a^{-j} \xi\right) e^{2 \pi i k \xi /\left(a^{j} c_{j}\right)} \mathrm{d} \xi\right|^{2}=\int_{\mathbb{R}}|\hat{f}(\xi)|^{2}\left|\hat{\psi}\left(a^{-j} \xi\right)\right|^{2} \mathrm{~d} \xi .
\end{aligned}
$$


The last step is a consequence of the fact that $\operatorname{supp} \hat{\psi}\left(a^{-j} \cdot\right) \subset\left(-a^{j}, a^{j}\right)$ and that $c_{j} \geq 2$. Combining this with (6.7) yields

$$
\begin{aligned}
\left\|S_{\wedge}^{q} f\right\|^{2}= & \sum_{j=-J_{1}}^{J_{2}} \sum_{k \in \mathbb{Z}}\left|\left\langle f, D_{a^{j}} T_{k} \psi\right\rangle\right|^{2} \\
& +\left(\sum_{j<-J_{1}}+\sum_{j>J_{2}}\right) \int_{\left(-a^{j+2} \delta,-a^{j} \delta\right) \cup\left(a^{j} \delta, a^{j+2} \delta\right)}|\hat{f}(\xi)|^{2} \mathrm{~d} \xi \\
\geq & \sum_{j=-J_{1}}^{J_{2}}\left|\left\langle D_{a^{-j}} f, T_{k} \psi\right\rangle\right|^{2}+\left(\int_{|\xi|<a^{-J_{1}+1} \delta}+\int_{|\xi|>a^{J_{2}+1} \delta}\right)|\hat{f}(\xi)|^{2} \mathrm{~d} \xi .
\end{aligned}
$$

By (6.2),

$$
\begin{aligned}
& \text { (6.10) } \sum_{k \in \mathbb{Z}}\left|\left\langle D_{a^{-j}} f, T_{k} \psi\right\rangle\right|^{2} \\
& =a^{j} \int_{K_{\delta}}\left|\hat{f}\left(a^{j}(\xi-1)\right)+\hat{f}\left(a^{j} \xi\right)\right|^{2} \mathrm{~d} \xi+a^{j} \int_{\delta}^{1-a^{2} \delta}\left|\hat{f}\left(a^{j} \xi\right)\right|^{2} \mathrm{~d} \xi+a^{j} \int_{a^{2} \delta}^{1-\delta}\left|\hat{f}\left(a^{j}(\xi-1)\right)\right|^{2} \mathrm{~d} \xi \\
& =\int_{a^{j} K_{\delta}}\left|\hat{f}\left(\xi-a^{j}\right)+\hat{f}(\xi)\right|^{2} \mathrm{~d} \xi+\int_{a^{j} \delta<|\xi|<a^{j}\left(1-a^{2} \delta\right)}|\hat{f}(\xi)|^{2} \mathrm{~d} \xi .
\end{aligned}
$$

We claim that for all $j \in \mathbb{Z}$,

$$
I_{j} \leq 2 I_{j+1}+2 \int_{a^{j} K_{\delta}}\left|\hat{f}\left(\xi-a^{j}\right)+\hat{f}(\xi)\right|^{2} \mathrm{~d} \xi+\int_{a^{j} \delta<|\xi|<a^{j}\left(1-a^{2} \delta\right)}|\hat{f}(\xi)|^{2} \mathrm{~d} \xi,
$$

where $I_{j}:=\int_{a^{j} \delta<|\xi|<a^{j+1} \delta}|\hat{f}(\xi)|^{2} \mathrm{~d} \xi$.

Indeed, by $a^{j}(a \delta-1)<-a^{j+1} \delta$, we have that

$$
\begin{aligned}
\int_{a^{j}\left(1-a^{2} \delta\right)}^{a^{j+1} \delta}|\hat{f}(\xi)|^{2} \mathrm{~d} \xi & \leq 2 \int_{a^{j}\left(1-a^{2} \delta\right)}^{a^{j+1} \delta}\left(\left|\hat{f}\left(\xi-a^{j}\right)\right|^{2}+\left|\hat{f}\left(\xi-a^{j}\right)+\hat{f}(\xi)\right|^{2}\right) \mathrm{d} \xi \\
& \leq 2 \int_{a^{j}\left(1-a^{2} \delta\right)}^{a^{j+1} \delta}\left|\hat{f}\left(\xi-a^{j}\right)+\hat{f}(\xi)\right|^{2} \mathrm{~d} \xi+2 \int_{-a^{j+2} \delta}^{-a^{j+1} \delta}|\hat{f}(\xi)|^{2} \mathrm{~d} \xi .
\end{aligned}
$$

Likewise,

$$
\begin{aligned}
\int_{-a^{j+1} \delta}^{-a^{j}\left(1-a^{2} \delta\right)}|\hat{f}(\xi)|^{2} \mathrm{~d} \xi & \leq 2 \int_{-a^{j+1} \delta}^{-a^{j}\left(1-a^{2} \delta\right)}\left(\left|\hat{f}\left(\xi+a^{j}\right)\right|^{2}+\left|\hat{f}\left(\xi+a^{j}\right)+\hat{f}(\xi)\right|^{2}\right) \mathrm{d} \xi \\
& \leq 2 \int_{a^{j+1} \delta}^{a^{j+2} \delta}\left|\hat{f}\left(\xi-a^{j}\right)+\hat{f}(\xi)\right|^{2} \mathrm{~d} \xi+2 \int_{a^{j+1} \delta}^{a^{j+2} \delta}|\hat{f}(\xi)|^{2} \mathrm{~d} \xi
\end{aligned}
$$

Adding the last two inequalities yields

$$
\int_{a^{j}\left(1-a^{2} \delta\right)<|\xi|<a^{j+1} \delta}|\hat{f}(\xi)|^{2} \mathrm{~d} \xi \leq 2 I_{j+1}+2 \int_{a^{j} K_{\delta}}\left|\hat{f}\left(\xi-a^{j}\right)+\hat{f}(\xi)\right|^{2} \mathrm{~d} \xi,
$$

which, together with $\delta<1-a^{2} \delta<a \delta$, shows (6.11).

Take any $f \in L^{2}(\mathbb{R})$ with $\|f\|=1$ and let $\eta=\left\|S_{\Lambda}^{q}(f)\right\|^{2}$. By (6.9)

$$
\int_{Z}|\hat{f}|^{2} \leq \eta, \quad \text { where } Z=\left\{\xi:|\xi|<a^{-J_{1}+1} \delta\right\} \cup\left\{\xi:|\xi|>a^{J_{2}+1} \delta\right\} .
$$


On the other hand, combining (6.9), 6.10), 6.11) shows that

$$
I_{j} \leq 2\left(I_{j+1}+\eta\right) \quad \text { for }-J_{1} \leq j \leq J_{2} \text {. }
$$

By (6.12) we also have that $I_{J_{2}+1} \leq \eta$. Therefore, $I_{j} \leq 3 \cdot 2^{J_{2}-j} \eta$ for $-J_{1} \leq j \leq J_{2}$. Consequently,

$$
\|f\|^{2}=\int_{Z}|\hat{f}(\xi)|^{2} \mathrm{~d} \xi+\sum_{j=-J_{1}+1}^{J_{2}} I_{j} \leq 3 \cdot 2^{J_{1}+J_{2}} \eta .
$$

This proves that the frame operator $S_{\Lambda}^{q}$ of $\mathcal{A}_{\Lambda}^{q}(\psi)$ is bounded from below by a constant depending only on $J_{1}$ and $J_{2}$, thus completing the proof of Theorem 6.1

Remark 9. By Theorem 3.8, the frame bounds of the quasi-affine systems $\mathcal{A}_{\Lambda}^{q}(\psi)$ are not uniform for all $\Lambda \subset \mathbb{Z}$. More precisely, the lower frame bound of $\mathcal{A}_{\Lambda}^{q}(\psi)$ must approach 0 for some choice of sparser and sparser lattices $\Lambda$. By analyzing the proof of Theorem 6.1 it is not difficult to show that this happens for the family of lattices $\Lambda_{J}=(p q)^{J} \mathbb{Z}$ as $J \rightarrow \infty$. This is due to the fact that in this case the quasi-affine system $\mathcal{A}_{\Lambda}^{q}(\psi)$ coincides with the affine system $\mathcal{A}(\psi)$ at the scales $-J \leq j \leq J$ and that the same argument as in the first part of the proof of Theorem 6.1 applies.

Theorem 6.1 says that the lower frame bound is not preserved in general when we move from a quasi-affine system $\mathcal{A}_{\Lambda}^{q}(\Psi)$ to the corresponding affine system $\mathcal{A}(\Psi)$ for rational non-integer dilations. It is not known whether the same could happen with the upper bound. This leads to the following open problem.

Question 1. Let $\Psi \subset L^{2}\left(\mathbb{R}^{n}\right)$ and $A \in G L_{n}(\mathbb{Q})$. Suppose that $\mathcal{A}_{\Lambda_{0}}^{q}(\Psi)$ is a Bessel sequence for some oversampling lattice $\Lambda_{0} \subset \mathbb{Z}^{n}$. Is $\mathcal{A}(\Psi)$ necessarily a Bessel sequence?

\section{REFERENCES}

[1] M. Bownik, The structure of shift-invariant subspaces of $L^{2}\left(\mathbb{R}^{n}\right)$, J. Funct. Anal. 177 (2000), 282-309. MR1795633 (2001k:42037)

[2] M. Bownik, A characterization of affine dual frames in $L^{2}\left(\mathbb{R}^{n}\right)$, Appl. Comput. Harmon. Anal. 8 (2000), 203-221. MR.1743536 (2001d:42019)

[3] M. Bownik, Quasi-affine systems and the Calderón condition, Harmonic analysis at Mount Holyoke (South Hadley, MA, 2001), 29-43, Contemp. Math., 320, Amer. Math. Soc., Providence, RI, 2003. MR1979930 (2004h:42039)

[4] M. Bownik, Z. Rzeszotnik, The spectral function of shift-invariant spaces on general lattices, Wavelets, frames and operator theory, 49-59, Contemp. Math., 345, Amer. Math. Soc., Providence, RI, 2004. MR2066821 (2005e:42106)

[5] M. Bownik, E. Weber, Affine frames, GMRA's, and the canonical dual, Studia Math. 159 (2003), 453-479. MR2052234 (2005f:42077)

[6] J.W.S. Cassels, An introduction to the geometry of numbers, Classics in Mathematics, Springer-Verlag, Berlin, 1997. MR1434478 (97i:11074)

[7] O. Christensen, An introduction to frames and Riesz bases, Birkhäuser Boston Inc., Boston, MA, 2003. MR.1946982 (2003k:42001)

[8] C.K. Chui, W. Czaja, M. Maggioni, G. Weiss, Characterization of general tight wavelet frames with matrix dilations and tightness preserving oversampling, J. Fourier Anal. Appl. 8 (2002), 173-200. MR 1891728 (2003a:42038)

[9] C.K. Chui, X.L. Shi, J. Stöckler, Affine frames, quasi-affine frames, and their duals, Adv. Comput. Math. 8 (1998), 1-17. MR1607452 (99b:42037)

[10] A. Cohen, I. Daubechies, J.-C. Feauveau, Biorthogonal bases of compactly supported wavelets, Comm. Pure Appl. Math. 45 (1992), 485-560. MR1162365 (93e:42044) 
[11] M. Frazier, G. Garrigós, K. Wang, G. Weiss, A characterization of functions that generate wavelet and related expansion, Proceedings of the conference dedicated to Professor Miguel de Guzmán (El Escorial, 1996), J. Fourier Anal. Appl. 3 (1997), 883-906. MR.1600215 (99c:42058)

[12] D. Han, D. Larson, Frames, bases and group representations, Mem. Amer. Math. Soc. 147 (2000), no. 697. MR1686653 (2001a:47013)

[13] E. Hernández, D. Labate, G. Weiss, A unified characterization of reproducing systems generated by a finite family. II, J. Geom. Anal. 12 (2002), 615-662. MR1916862 (2003j:42036)

[14] E. Hernández, D. Labate, G. Weiss, E. Wilson, Oversampling, quasi-affine frames, and wave packets, Appl. Comput. Harmon. Anal. 16 (2004), 111-147. MR2038268 (2005a:42028)

[15] E. Hewitt, K. Ross, Abstract harmonic analysis. Vol. I: Structure of topological groups. Integration theory, group representations, Academic Press Inc., Publishers, New York, 1963.

[16] M. Holschneider, Wavelets. An Analysis Tool, Clarendon Press, Oxford, 1995. MR1367088 (97b:42051)

[17] R.S. Laugesen, Completeness of orthonormal wavelet systems for arbitrary real dilations, Appl. Comput. Harmon. Anal. 11 (2001), 455-473. MR.1866351 (2002h:42073)

[18] R.S. Laugesen, Translational averaging for completeness, characterization and oversampling of wavelets, Collect. Math. 53 (2002), 211-249. MR.1940326 (2003i:42053)

[19] A. Ron, Z. Shen, Frames and stable bases for shift-invariant subspaces of $L_{2}\left(\mathbb{R}^{d}\right)$, Canad. J. Math. 47 (1995), 1051-1094. MR1350650 (96k:42049)

[20] A. Ron, Z. Shen, Affine systems in $L_{2}\left(\mathbb{R}^{d}\right)$ : the analysis of the analysis operator, J. Funct. Anal. 148 (1997), 408-447. MR1469348 (99g:42043)

[21] A. Ron, Z. Shen, Weyl-Heisenberg frames and Riesz bases in $L_{2}\left(\mathbb{R}^{d}\right)$, Duke Math. J. 89 (1997), 237-282. MR1460623 (98i:42013)

[22] A. Ron, Z. Shen, Affine systems in $L_{2}\left(\mathbb{R}^{d}\right)$. II. Dual systems, J. Fourier Anal. Appl. 3 (1997), 617-637. MR:1491938 (99g:42044)

[23] A. Ron, Z. Shen, Generalized shift-invariant systems, Constr. Approx. 22 (2005), 1-45. MR2132766 (2006c:42030)

Department of Mathematics, University of Oregon, Eugene, Oregon 97403-1222

E-mail address: mbownik@uoregon.edu

Department of Mathematics, Technical University of Denmark, Matematiktorvet, Building 303S, DK-2800 Kgs. Lyngby, Denmark

E-mail address: J.Lemvig@mat.dtu.dk

Current address: Institut für Mathematik, Universität Osnabrück, 49069 Osnabrück, Germany

E-mail address: jlemvig@uni-osnabrueck.de 